Notfall Rettungsmed 2010 · 13:723-736

DOI 10.1007/s10049-010-1375-y

Online publiziert: 16.11 .2010

(c) European Resuscitation Council 2010

J. Soar ${ }^{1} \cdot$ K. Monsieurs ${ }^{2} \cdot$ J. Ballance ${ }^{3} \cdot$ A. Barelli ${ }^{4} \cdot$ D. Biarent ${ }^{5} \cdot$ R. Greif ${ }^{6} \cdot$ A. Handley ${ }^{7}$.

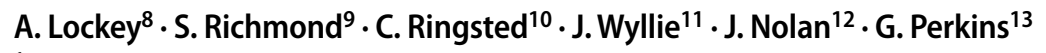

1 Southmead Hospital, North Bristol NHS Trust Bristol

${ }^{2}$ Ghent University Hospital, Gent

${ }^{3}$ Orchid Bank, Woolhope, Herefordshire

${ }^{4}$ Department of Clinical Toxicology - Poison Centre and Emergency Department, Catholic University School of Medicine, Rom

5 Paediatrics, Paediatric Intensive Care and Emergency Medicine, Libre de Bruxelles, Queen Fabiola Children's University Hospital Brussels, Brüssel

${ }^{6}$ Dept. Anesthesiology and Pain Therapy, Peripheral Anesthesia Division, Medical Education Program, University Hospital Bern, Inselspital, Bern

${ }^{7}$ Colchester

${ }^{8}$ Calderdale and Huddersfield NHS Trust, Salterhebble, Halifax

${ }^{9}$ Sunderland Royal Hospital, Sunderland

${ }^{10}$ Centre for Clinical Education, University of Copenhagen and Capital Region, Denmark, Rigshospitalet, Kopenhagen

${ }^{11}$ James Cook University Hospital, Middlesbrough

12 Royal United Hospital, Bath

${ }^{13}$ University of Warwick, Warwick Medical School, Warwick

\title{
Unterrichtsprinzipien zur Wiederbelebung
}

\section{Sektion 9 der Leitlinien zur Reanimation 2010 des European Resuscitation Council}

\section{Einführung}

Das Überleben eines Kreislaufstillstands wird von der Qualität der den Leitlinien zugrunde gelegten wissenschaftlichen Evidenz, der Effektivität der Ausbildung der Helfer und den Ressourcen für die Umsetzung der Leitlinien bestimmt [1]. Weitere Einflussfaktoren sind die Bereitschaft der Helfer zur Umsetzung der Leitlinien in die Praxis und die „human factors“, die die Steuerung, Überwachung und Nutzung technischer Systeme durch den Menschen ermöglichen [2]. Die Leitlinien 2010 werden wahrscheinlich mit einer sorgfältig geplanten umfassenden Einführungsstrategie, die die Ausbildung einschließt, schneller umgesetzt. Verspätet verfügbares Trainingsmaterial und eine mangelnde Zahl an Trainern wurden als Gründe für die Verzögerungen in der Implementierung der Leitlinien 2005 genannt $[3,4]$.

Die vorliegende Sektion beschreibt die Schlüsselelemente des Unterrichts, die vom International Liaison Committee on Resuscitation (ILCOR; [5]) durch Evaluation der Evidenz identifiziert wurden. Es werden die wissenschaftlichen Grundlagen der Ausbildung in „basic life support“ (BLS) und „advanced life support“ (ALS) erörtert sowie eine Aktualisierung der Kurse des European Resuscitation Council (ERC; [6]) vorgestellt.

\section{Schlüsselempfehlungen für den Unterricht}

Die Schlüsselfragen, die durch die ILCORArbeitsgruppe Ausbildung, Implementierung und Teams (Education, Implementation and Teams; EIT) im Rahmen der Bewertung der Evidenz beim Prozess der Erstellung der Leitlinien 2010 als relevant für diese Sektion [5] ermittelt wurden, sind:

- Ausbildungsmethoden sollen evaluiert werden, um sicherzustellen, dass die Lernziele verlässlich erreicht werden. Ziel ist es, zu gewährleisten, dass die Lernenden die notwendigen Fertigkeiten und das notwendige Wissen erwerben und behalten, um bei einem Kreislaufstillstand korrekt handeln und das Outcome des Patienten verbessern zu können.

- Kurze Video-/Computerkurse zum Eigenstudium mit oder ohne minimale Anleitung durch einen Instruktor werden, kombiniert mit „Hands-on“Übungen, als effektive Alternative zu traditionellen BLS-Instruktorkursen [Themen: „cardiopulmonary resuscitation“ (CPR) und automatisierte externe Defibrillation (AED)] betrachtet.

- Idealerweise sollen alle Bürger in Standard-CPR-Methoden (Herzdruckmassage und Beatmung) ausgebildet werden. Unter bestimmten Umständen ist jedoch das ausschließliche Training in der Herzdruckmassage sinnvoll („chest compression-only CPR“, z. B. gelegentliches Training bei sehr begrenzter Zeit). Wer nur in der 
Herzdruckmassage ausgebildet ist, soll angehalten werden, Standard-CPRMaßnahmen zu erlernen.

- Die Kenntnisse und Fertigkeiten der BLS- und ALS-Techniken nehmen in nur 3 bis 6 Monaten ab. Häufige Überprüfung zeigt, wer ein Auffrischungstraining benötigt, um seine Kenntnisse und Fertigkeiten zu erhalten.

- Hilfsmittel, die CPR-Kommandos oder -Rückmeldung geben, verbessern das Erlernen und Behalten von CPRFertigkeiten. Sie sollen für das CPRTraining von Laien- und professionellen Helfern berücksichtigt werden.

- Die Gewichtung von nichttechnischen Fähigkeiten wie Führungsqualität, Teamfähigkeit, Prozesssteuerung und strukturierte Kommunikation trägt dazu bei, die CPR-Qualität und die Patientenversorgung zu verbessern.

- Teambesprechungen, um CPR vorab zu planen („briefing“), und Nachbesprechungen nach simulierten oder realen CPR-Versuchen („debriefing“) sollen zur Verbesserung der Teamleistung und der individuellen Fähigkeiten genutzt werden.

- Forschungsergebnisse zum Einfluss des Reanimationstrainings auf das tatsächliche Patienten-Outcome sind sehr begrenzt. Obgleich Studien an Übungsphantomen sinnvoll sind, sollen Wissenschaftler angehalten werden, den Einfluss der Ausbildungsmethoden auf das wahre Patienten-Outcome zu untersuchen und darzustellen.

\section{Wen und wie trainieren}

Idealerweise sollen alle Bürger die Maßnahmen der Wiederbelebung kennen. Es gibt keine ausreichende Evidenz für oder gegen Trainingsmaßnahmen, die auf spezielle Hochrisikopopulationen zielen. Trotzdem kann ein Training die Angst von Patienten und Angehörigen reduzieren; es verbessert ihre emotionale Einstellung und vermittelt ihnen das Gefühl, handlungsfähig zu sein [5].

Das Training in CPR-Maßnahmen wird von vielen Menschen benötigt, von Laien, Bürgern ohne medizinische Ausbildung, aber mit einer Aufgabe, die sie in die Garantenstellung bringt (z. B. Rettungsschwimmer, Erste-Hilfe-Personal) bis hin zu pro- fessionellen Helfern im Rettungswesen, auf den Normalstationen im Krankenhaus und in den Intensivpflegeeinrichtungen.

Das Training soll auf die Bedürfnisse der unterschiedlichen Lerntypen und -stile zugeschnitten sein, um das Erwerben und Behalten von Wissen sowie Fertigkeiten der Wiederbelebung sicherzustellen. Wer regelmäßig Reanimationen durchführt, muss die aktuellen Leitlinien kennen und fähig sein, diese in einem multiprofessionellen Team effektiv auszuführen. Diese Personen benötigen ein komplexeres Training, das technische und nichttechnische Fertigkeiten kombiniert (z. B. Teamarbeit, Führungsqualität, strukturierte Kommunikation; [7, 8]). Im folgenden Abschnitt haben die Autoren diese willkürlich in Basisund Fortgeschrittenenniveau unterteilt; in der Wirklichkeit stellen sie ein Kontinuum dar. Die meisten Forschungsergebnisse in diesem Bereich beruhen auf dem Training in der Erwachsenenreanimation. Viele dieser Forschungsergebnisse können auch auf das Training der Kinder- und Neugeborenenwiederbelebung übertragen werden.

\section{Basistraining und Training in automatisierter externer Defibrillation}

Wiederbelebung und Frühdefibrillation durch Notfallhelfer retten Leben. Viele Faktoren können jedoch die Bereitschaft von Notfallhelfern verringern, die Wiederbelebung zu beginnen. Dazu gehören Panik, Angst vor Krankheiten oder davor, den Patienten zu verletzen, aber auch die Wiederbelebung nicht korrekt auszuführen $[9,10,11,12,13,14,15,16,17,18,19,20$, 21, 22, 23, 24]. Wiederbelebungstraining von Laien erhöht die Bereitschaft, Hilfe zu leisten $[12,18,19,20,25,26,27,28,29,30]$.

Das Wiederbelebungstraining und die Wiederbelebung eines konkreten Patienten sind meistens sicher. Teilnehmer an Wiederbelebungskursen müssen auf das Ausmaß der körperlichen Leistung, das erforderlich ist, hingewiesen werden. Helfer, die bedrohliche Symptome (Thoraxschmerzen, Kurzatmigkeit) während eines Trainings entwickeln, müssen dieses beenden. Helfer, die während einer realen Wiederbelebung ernsthafte Symptome entwickeln, sollen das Beenden der Maßnahmen erwägen (s. Sektion 2 „Lebens- rettende Basismaßnahmen für Erwachsene und Verwendung automatisierter externer Defibrillatoren“; [31]).

\section{Curriculum}

Das Curriculum von BLS- und AEDKursen soll auf die Zielgruppe zugeschnitten sein und so einfach wie möglich gehalten werden. Folgende Kernelemente sind zu beachten $[5,32]$ :

- persönliches und Umgebungsrisiko vor dem Beginn der CPR-Maßnahmen,

- Erkennen des Kreislaufstillstands durch Beurteilen der Ansprechbarkeit des Patienten, Öffnen seiner Atemwege und Beurteilen seiner Atmung [31, 32].

- Erkennen von Schnappatmung oder nichtnormaler Atmung als Zeichen des Kreislaufstillstands bei bewusstlosen Menschen [33, 34],

- Thoraxkompressionen von hoher Qualität (Beibehalten der Kompressionsfrequenz, der Eindrücktiefe und der Entlastung des Brustkorbs, minimale Hands-off-Zeiten) und Beatmung,

- Benutzerführung/Feedback (einschließlich Geräte) während der CPR sollen auch im BLS-Training zur Verbesserung des Erlernens und zum Erhalt der Fertigkeiten beitragen [35].

- Jedes BLS-/AED-Training soll die Standard-CPR (einschließlich Beatmung/Ventilation) zum Ziel haben. Das ausschließliche Training der Thoraxkompression im Vergleich zum Training von Thoraxkompressionen in Verbindung mit der Beatmung kann in speziellen Situationen Vorteile haben $[10,15,18,23,24,27,36,37]$. Ein Ansatz dazu wird unten beschrieben.

\section{Unterricht von Standardmaß- nahmen vs. Chest compression- only CPR}

Welche Fertigkeiten welchen Helfern vermittelt werden sollen, ist noch nicht geklärt. Die Beschränkung auf Thoraxkompression ist schneller und einfacher, speziell wenn viele Menschen unterrichtet werden sollen, die sonst keinen Zugang zu einem Wiederbelebungstraining haben. In vielen Situationen aber ist eine Standard-CPR (die Ventilation/Mund-zu- 
Mund-Beatmung einschließt) besser, so bei Kindern [38], Kreislaufstillstand durch Atemstillstand und wenn eine Wiederbelebung länger als ein paar Minuten erforderlich ist [32]. Deshalb wird folgender vereinfachter pädagogischer Ansatz vorgeschlagen:

- Idealerweise werden alle Bürger in Standard-CPR-Maßnahmen [Thoraxkompressionen und Beatmungen im Kompression-Ventilation- (CV-)Verhältnis von 30:2] ausgebildet.

- Wenn die verfügbare Zeit für die Ausbildung eingeschränkt ist oder die Ausbildung opportunistisch (Telefoninstruktion eines Notfallzeugen durch einen Leitstellendisponenten, Massenveranstaltungen, Werbekampagnen, „YouTube viral videos“ oder wenn eine Person nicht ausgebildet werden will) erfolgt, soll sich das Training auf die Vermittlung von Thoraxkompressionen reduzieren.

- Wer nur in Thoraxkompressionen ausgebildet wurde, soll weiteres Training in Beatmung (und Thoraxkompressionen) erhalten; idealerweise wird es ihm gleich in derselben Unterrichtseinheit angeboten.

- Laien mit Garantenstellung (Erste-Hilfe-Personal, Rettungsschwimmer, Kindergärtner etc.) sollen in der Durchführung von Thoraxkompressionen und Beatmungen ausgebildet werden.

- Bei Kindern sollen Helfer ermutigt werden, das CV-Verhältnis anzuwenden, das sie in der Erwachsenenreanimation gelernt haben, da das Outcome des kindlichen Patienten schlechter ist, wenn der Notfallhelfer jegliche Maßnahmen unterlässt. Laienhelfer, die die Wiederbelebung von Kindern erlernen wollen, weil sie Verantwortung für Kinder haben (Eltern, Lehrer, Pflegedienstpersonal, Rettungsschwimmer), soll vermittelt werden, dass es vorzuziehen ist, den Erwachsenenablauf zu modifizieren, indem sie die CPR mit 5 Beatmungen beginnen und danach ungefähr eine 1 min lang CPR-Maßnahmen durchführen, bevor sie Hilfe holen gehen, wenn niemand anderes das übernehmen kann. Die notwendige Tiefe qualitativ ausreichender Thorax- kompressionen bei Kindern beträgt ein Drittel des Thoraxdurchmessers [39].

Das Training von CPR-Maßnahmen aller Bürger soll gefördert werden. Fehlendes Training darf aber kein Hindernis für die Wiederbelebung durch Chest compression-only CPR sein, die vorzugsweise auf telefonische Anweisung des Leitstellendisponenten vorgenommen werden soll.

\section{Methoden}

Es gibt zahlreiche Formen des BLS-/AEDUnterrichts. Am häufigsten wird der traditionelle, vom Instruktor geleitete Kurs durchgeführt [40]. Verglichen zu diesen traditionellen Kursen sind gut gestaltete Selbstlernprogramme (Video, DVD, Computer) mit minimalem oder ohne Instruktoreinsatz eine effektive Alternative zum Erlernen der BLS-/AED-Fertigkeiten für Laien- und professionelle Helfer $[41,42,43,44,45,46,47,48,49,50,51,52$, $53,54,55]$. Essenziell für diese Kurse sind praktische Übungen.

Der Einsatz eines AED durch einen Notfallhelfer ohne vorhergehendes offizielles Training kann nutzbringend und lebensrettend sein $[45,56,57,58,59,60]$. Die Anwendung des AED (schnelle Vorbereitung, korrektes Platzieren der Elektroden) kann durch kurzes Training von Laien- und professionellen Helfern verbessert werden $[45,50,61,62]$.

\section{Dauer und Frequenz der instruktorgeführten Trainingskurse}

Die optimale Dauer instruktorgeleiteter BLS-Kurse wurde noch nicht festgelegt; sie ist vermutlich von den Kursteilnehmern (Laien-, professionelle Helfer, Vorkenntnisse oder Alter der Teilnehmer), vom Curriculum, vom Zahlenverhältnis der Instruktoren zu den Teilnehmern, der Intensität des Hands-on-Trainings und der Überprüfung des Lernerfolgs am Abschluss des Kurses abhängig.

Die meisten Studien zeigen, dass die CPR-Fertigkeiten (Absetzen des Notrufs, Thoraxkompressionen, Beatmungen) innerhalb von 3 bis 6 Monaten nach dem durchgeführten Training abnehmen [43, $46,63,64,65,66,67,68]$. Die Fertigkeiten im Umgang mit dem AED werden länger behalten als die BLS-Fertigkeiten allein [59, $64,96]$. Reevaluation, ein „Refresher“-Kurs oder erneutes Training nach nur 3 bis 6 Monaten erhalten oder verbessern die Qualität der CPR-Maßnahmen [64, 70, 71, 72, 73].

\section{Verwendung von \\ Benutzerführung/Feedbackgeräten}

Benutzerführung und Feedbackgeräte sollen in das Training von Laien- und professionellen Helfern eingebunden werden [35]. Die Geräte können durch die Reanimation führen (Signale zur Durchführung einer Maßnahme, Sprachanweisungen, Messung von Tiefe und Frequenz der Thoraxkompressionen), eine Rückmeldung z. B. über eine visuelle Anzeige der Kompressionstiefe geben oder über eine Kombination aus Anweisungen und Feedback verfügen. Die Ausbildung mithilfe solcher Systeme verbessert die Ausführung der Wiederbelebung sowie das Erwerben und Behalten der Fertigkeiten. Studien haben den Effekt an einem Übungsphantom ohne solche Hilfen gemessen $[63,74,75,76,77,78]$. Es ist zu beachten, dass automatische Feedback-Mechanismen die Eindrücktiefe der Thoraxkompressionen überschätzen, wenn der Patient auf einer weichen, elastischen Unterlage, z. B. eine Matratze, liegt [79, 8o].

\section{Training für Fortgeschrittene}

\section{Curriculum}

Fortgeschrittenes Training (in ALS-Maßnahmen) findet in der Regel für professionelle Helfer statt. Das Curriculum soll auf die individuellen Bedürfnisse der Lernenden zugeschnitten sein, auf die Vielzahl verschiedener Patienten eingehen und die Rolle der professionellen Helfer innerhalb des Versorgungssystems für Patienten nach Kreislaufstillstand definieren. Die Evidenz $\mathrm{zu}$ spezifischen Interventionen in ALSKursen, die das Lernen und Behalten der Fähigkeiten verbessern, ist limitiert. Ein nach den Leitlinien 2005 absolvierter ALSKurs des ERC ging in einer Studie mit simuliertem Kreislaufstillstand mit einer Reduktion in der "No-flow“-Zeit einher, verbesserte aber keine anderen Qualitätsparameter [81]. Größere klinische Erfahrung der Lernenden scheint den Langzeiterhalt 
von Wissen und Fertigkeiten zu verbessern $[82,83]$.

Studien zur Anwendung des ALS bei realem oder simuliertem innerklinischen Kreislaufstillstand [84, 85, 86, 87, 88, 89, 90, 91, 92, 93, 94] belegten eine verbesserte Teamleistung, wenn ein spezifisches Team- und/oder Führungstraining im Rahmen des Standard-ALS-Kurses stattgefunden hatte. Teamtraining und die Fähigkeit zur Herzrhythmuserkennung sind essenziell, um die Hands-off-Zeit zu minimieren, wenn die Strategie der manuellen Defibrillation der 2010er Leitlinien verfolgt wird [95, 96].

Kernelemente für ein ALS-Kurs-Curriculum sollen folgende Themen beinhalten:

- Prävention von Kreislaufstillstand [97, 98],

- Durchführung qualitativ hochwertiger

Thoraxkompressionen (Kompressi-

onsfrequenz, -tiefe, Entlastung des

Thorax, minimale „Hands-off“-Zeit,

Ventilation mit Taschenmaske, Beutel-

Maske-Beatmung),

- Defibrillation, einschließlich des Ladens des Defibrillators während der Thoraxkompression,

- ALS-Algorithmus,

- nichttechnische Fertigkeiten (z. B. Führungseigenschaften, Teamtraining, strukturierte Kommunikation).

Ein erweitertes Training kann Themen $\mathrm{zu}$ fortgeschrittenem Atemwegsmanagement, Management von „Peri-arrest“-Arrhythmien, Wiederbelebung unter speziellen Umständen, Gefäßzugang, Medikamente in der Wiederbelebung, Reanimationsnachsorge und Ethik der Wiederbelebung beinhalten.

\section{Methoden}

\section{Vorbereitendes Training}

Vielfältige Methoden können zur Vorbereitung der Teilnehmer auf einen Wiederbelebungskurs eingesetzt werden (Lesen der Unterrichtsmaterialien, Test vor dem Kurs, E-Learning; [99, 100, 101, 102, 103, 104, 105, 106, 107]). Eine aktuelle große randomisierte kontrollierte Studie eines kommerziell erhältlichen E-Learning-Simulationsprogramms vor Teilnahme an einem ALS-Kurs im Vergleich zur Standardvorbereitung mit dem Kursmanual zeigte keine Verbesserung in kognitiven und psychomotorischen Fertigkeiten bei Tests am simulierten Kreislaufstillstand $[107,108]$.

Zahlreiche andere Studien zu alternativen Unterrichtsmethoden erheben den Anspruch, dass computer- oder videobasiertes Training gleich oder besser ist oder den Trainerzeitaufwand reduziert [100, 101, 106, 109, 110, 111, 112, 113, 114, 115, 116, $117,118,119,120,121,122,123]$. Jede Vorbereitungsmethode auf den Kurs, die Verbesserung im Wissen und in den Fertigkeiten oder eine Reduktion der Trainerpräsenzzeit anstrebt, soll offiziell evaluiert werden, um die Äquivalenz oder Verbesserung im Vergleich zur Standardausbildung sicherzustellen. Eine weitere multizentrische randomisierte kontrollierte Studie überprüft, ob ein Eintages-ALS-Kurs mit E-LearningZusatzmaterial einem Zweitagestandardkurs gleichkommt (ISRCTN86380392).

\section{Simulation und Training der realistischen Wiederbelebung}

Simulation ist ein essenzieller Teil der Reanimationsausbildung. Die Bandbreite für die Anwendung der Simulation in der Reanimationsausbildung ist groß [124]. Ein Mangel an einheitlichen Definitionen, (z.B. „high" vs. „low fidelity simulation“) macht den Vergleich unterschiedlicher Studien schwierig. Simulationstraining ging ziemlich konsistent [33, 125, 126, 127, 128, 129, 130, 131, 132, 133, 134, 135, 136], aber nicht generell [137, 138, 139, 140, 141, 142, 143] mit einer Verbesserung im Wissen und in der Geschicklichkeit der Durchführung der CPR an Übungsphantomen einher. Limitiert ist dagegen die Evidenz der Übertragbarkeit auf das reale Leben. Wenige Vorher-nachher-Studien über den Effekt des Wiederbelebungstrainings mithilfe der Simulation auf die tatsächliche reale Durchführung belegten Verbesserungen des Patienten-Outcome [144, 145, $146,147,148]$. In diesen Studien lässt sich aber der Effekt des Simulationstrainings nicht von anderen temporären Faktoren des Unterrichts unterscheiden. Eine randomisierte kontrollierte Studie und eine prospektive fallkontrollierte Studie demonstrierten eine verbesserte Durchführung bei Vorhandensein dieser Fertigkeiten (Vergleich Simulator- zu Standardtraining; [127, 149]).
Es existieren widersprüchliche Daten darüber, ob realistischere Bedingungen (naturgetreuere Übungsphantome, wirkliche Schauplätze) den Lernerfolg der Helfer und auch das Outcome der Patienten verbessern $[125,128,133,135,137,138,141,150,151,152,153$, 154]. Eine Studie zeigte einen signifikanten Wissenszuwachs bei der Verwendung von Übungsphantomen oder simulierenden Personen in der Traumaversorgung [153]. Es ergab sich aber kein Unterschied im Erwerb des Wissens zwischen der Verwendung von Übungsphantomen oder simulierenden Personen, obwohl die Lernenden die Übungsphantome bevorzugten.

Es ergibt sich eine ungenügende $\mathrm{Be}$ weislage für oder gegen den Einsatz realistischerer Bedingungen im BLS- und ALSUnterricht (z. B. lebensechte Übungsphantome oder In-situ-Training), um das Trainingsergebnis (z. B. Demonstration der Fähigkeiten am Übungsphantom oder in der realen CPR-Situation, Durchführungsbereitschaft) $\mathrm{zu}$ verbessern, wenn mit Standardtrainingsbedingungen verglichen wird. Die Auswirkungen auf das Kosten-Nutzen-Verhältnis bei Verwendung von „High-fidelity“-Simulatoren müssen noch untersucht werden [141].

Zukünftige Studien sollen den Effekt von Trainingsinterventionen (einschließlich der Simulation) auf das Patienten-Outcome und die reale Reanimation untersuchen. Die Auswertungen von Krankengeschichten [155], Qualitätssicherungsstudien [149] und Methoden zur Dokumentation der Qualität der Wiederbelebung [156] bestätigen die Machbarkeit solcher Ansätze.

\section{Trainingsintervalle}

Wissen und Fertigkeiten nehmen nach einem Wiederbelebungstraining rasch ab. Eine Wiederauffrischung ist erforderlich, um das Wissensniveau und die Fertigkeiten aufrechtzuerhalten. Bis jetzt ist das optimale Wiederholungsintervall nicht festgelegt. In den meisten Studien nahmen die Fertigkeiten und das Wissen über den ALS innerhalb von 3 bis 6 Monaten nach dem Training ab $[65,157,158,159,160,161,162,163$, 164], 2 Studien ermittelten einen Zeitraum zwischen 7 und 12 Monaten, $[165,166]$ und eine Studie verwies auf 18 Monate [167]. 


\section{Prüfungen}

Die beste Methode, den Wissenserwerb und den Lernfortschritt während des Kurses zu prüfen, ist nicht bekannt. Schriftliche Tests in ALS-Kursen können die Umsetzung in der Praxis nicht vorhersagen und sollen die Demonstration der klinischen Fertigkeiten nicht ersetzen $[166,167,168,169,170,171]$. Eine Prüfung am Kursende scheint einen positiven Effekt auf die Durchführung und Beibehaltung zu haben und soll berücksichtigt werden $[172,173]$.

\section{Alternative Strategien zur Verbesserung}

Verwendung von kognitiven Hilfsmittel (Checklisten usw.). Kognitive Hilfsmittel wie Checklisten können verwendet werden, um das Einhalten der Leitlinien zu verbessern, solange es dadurch nicht $\mathrm{zu}$ Verzögerungen im Beginn der Wiederbelebung kommt und die richtigen Checklisten verwendet werden $[174,175,176,177$, $178,179,180,181,182,183,184,185,186]$. Checklisten sollen vor ihrer Verwendung in Situationen der simulierten Wiederbelebung getestet werden $[84,85,86,87,88$, $89,90,91,92,93,94]$.

Übungsalarm. Übungsalarme und Übungseinsätze ermöglichen es, die Reaktion des einzelnen Helfers und des Notfallsystems auf den Patienten mit Kreislaufstillstand zu testen. Alarmübungen können das Wissen [187], die Durchführungsfertigkeiten [188], das Selbstvertrauen [189] und die Vertrautheit mit der Umgebung [190] der ALS-Helfer verbessern sowie Fehler im System und bei den Helfern identifizieren [191, 192].

"Briefing" und „debriefing" von Teams. Briefing und Debriefing sollen während des Trainings und bei klinischen Einsätzen zur Anwendung kommen. Erfolgreiche Teams (wie im Sport) treffen sich vor und nach jedem Einsatz. Umfragen in Großbritannien [193] und Kanada [194] haben ergeben, dass Notfallrettungsteams selten offizielle Briefing- und DebriefingTreffen haben. Debriefing und Feedback sind unterschiedlich, aber miteinander verwandt, da einzelne Formen des Feedbacks Bestandteil des Debriefing sind.
Debriefing ist „Face-to-face“-Kommunikation und bindet alle Beteiligten ein. Feedback gibt Informationen über beobachtete Ereignisse unter Verwendung verschiedener Methoden (Videoaufnahmen, Defibrillatoraufzeichnungen, geübte Beobachter). Debriefing scheint eine effektive Methode zur Verbesserung der CPR und möglicherweise auch des PatientenOutcome zu sein, solange objektivierbare Sachverhalte diskutiert werden [87, 89, 127, 149, 195, 196, 197, 198, 199, 200, 201, 202, 203, 204, 205]. Dennoch ist die ideale Form des Debriefing noch unklar.

\section{Reanimationskurse des European Resuscitation Council}

Der ERC hält ein breites Angebot an Trainingskursen mit dem Ziel vor, die Lernenden zubefähigen, unter realen Bedingungen CPR-Maßnahmen auf dem zu erwartenden Niveau ausführen zu können, ob dies nun Laien- oder Notfallhelfer im eigenen Lebensbereich oder im Krankenhaus oder professionelle Helfer (Rettungsdienst, allgemeine Station im Krankenhaus, Funktions- oder Intensivbereich, Notfallteam) sind.

Die ERC-Kurse fokussieren auf Unterricht in Kleingruppen mit interaktiven Diskussionen, Hands-on-Training praktischer Fertigkeiten und klinische Simulation an Übungsphantomen [6, 206]. Das günstige Zahlenverhältnis von Instruktoren zu Teilnehmern beträgt je nach Kursart 1:3 bis 1:6. Vollständige Information über die Kurse des ERC und deren Terminologie sind auf den Webseiten http:// www.erc.edu bzw. grc-org.de erhältlich.

\section{Philosophie}

Die Kurse werden von Instruktoren geleitet, die in Lehr- und Prüfungsmethoden ausgebildet sind. Das Unterrichtsklima soll so positiv gestaltet werden, dass das Lernen gefördert wird. Um mögliche Unterschiede zwischen Lehrenden und Lernenden abzubauen, wird die Anrede mit den Vornamen angestrebt. Die Interaktionen zwischen den Instruktoren und den Teilnehmern sollen positiv ablaufen; das Lernen wird durch Ermutigung mithilfe eines konstruktiven Feedbacks und eines Debriefing der Resultate geleitet. Das
Mentorensystem führt zu einem verbesserten Feedback und zur Unterstützung der Teilnehmer. Besonders während der Prüfungen ist ein gewisser Stress bei den Teilnehmern unvermeidbar [207], doch besteht das Ziel der Instruktoren darin, diese zu Bestleistungen anzuspornen.

\section{Management}

Die Kurse werden von Spezialistenkomitees innerhalb jedes National Resuscitation Council (NRC) und vom International Course Committee des ERC beaufsichtigt. Der ERC hat das webbasierte Course Management System (http://courses.erc. edu) entwickelt. Dieses System kann zur Registrierung in allen ERC-Kursen genutzt werden und ermöglicht Kursorganisatoren eines jeden Landes, einen Kurs anzumelden, Instruktoren auszuwählen, die Teilnehmeranwesenheit und den erfolgreichen Kursabschluss zu bestätigen. Kursdirektorberichte können mithilfe des Systems direkt an den ERC geschickt werden. Kursteilnehmer können sich direkt oder über den Kursorganisator registrieren lassen. Am Ende eines Kurses generiert das System die Kurszertifikate für die Teilnehmer und die Instruktoren. Diese Zertifikate haben eine sie kennzeichnende Nummer und können über die Kursorganisatoren oder -direktoren jederzeit abgerufen werden. Teilnehmer, die erfolgreich einen Kurs abgeschlossen haben, erwerben den „Provider“-Status. So wird z. B. ein Teilnehmer mit erfolgreich abgeschlossenem ALS-Kurs zum „ALS-Provider“. Die NRC haben Zugang zu Informationen über Kurse in ihren Ländern.

\section{Sprache}

Ursprünglich wurden die ERC-Kurse von einem Team aus internationalen Instruktoren in englischer Sprache abgehalten [206]. Seitdem jedoch in den verschiedenen Ländern genügend Instruktoren ausgebildet und die Kursunterlagen sowie -materialien in die einzelnen Sprachen übersetzt wurden, finden die Kurse zunehmend in der Muttersprache der Teilnehmer statt. Frühe Übersetzung der Leitlinien und des Kursmaterials ist entscheidend, um die Umsetzung der Erneuerungen nicht zu verzögern [3]. 


\section{Instruktoren}

Um geeignete Instruktoren zu identifizieren und sie zu trainieren, wurde eine erprobte Methode etabliert.

\section{Eignung}

Die Eignung zum Instruktor (potenzielle Instruktoren, IP) kann vom Instruktorteam den Teilnehmern ausgesprochen werden, die einen Kurs mit hoher Erfolgsquote abgeschlossen haben und während eines Provider-Kurses Sachkompetenz, besonders aber Führungsqualitäten, Teamfähigkeit und klinische Kompetenz zeigten. Dies beinhaltet auch, dass sie wortgewandt, hilfsbereit und motiviert sind. Diese besonderen Teilnehmer eines Provider-Kurses werden zu einem Instruktorenkurs eingeladen. Der "generic instructor course" (GIC) dient der Schulung zum Instruktor für folgende Kurse: „advanced life support“ (ALS), „European paediatric life support“ (EPLS), „newborn life support“ (NLS), „immediate life support“ (ILS) und „European pediatric immediate life support“ (EPILS). Für jene, die nur ERC-BLS/AED unterrichten wollen, gibt es einen speziellen BLS/AED-Instruktoren-Kurs.

\section{Kurse}

Die Instruktorkurse werden von erfahrenen Instruktoren durchgeführt, im Fall des GIC unter Einbindung eines Pädagogen, der in besonderem Maß mit der Vermittlung medizinischer Kenntnisse und den Prinzipien der Erwachsenenbildung vertraut ist. (Details dieser Instruktorenkurse sind unten angeführt.) Die Bewertungsmethode wird vom Fachbereich geprägt und bei Bedarf ein entsprechendes Feedback gegeben.

\section{Kandidaten}

Wer einen Instruktorkurs erfolgreich abgeschlossen hat, wird zum Instruktorkandidaten (,instructor candidate“,IC), nachfolgend 2 getrennte Kurse unter Supervision unterrichten und während der Kurse konstruktives Feedback zu der erbrachten Leistung erhalten. Nach 2 erfolgreichen Kursen steigt der ICzum „full instructor“ auf. Gelegentlich kann das Instruktorteam jedoch entscheiden, dass noch ein weiterer Kurs notwendig ist, oder in seltenen Fällen, dass ein IC nicht geeignet ist, Full instructor zu werden. Da- gegen kann beim zuständigen internationalen Kurskomitee Einspruch erhoben werden; dort fällt die endgültige Entscheidung.

\section{Kursdirektor}

Jeder ERC-Kurs steht unter der Leitung eines anerkannten Kursdirektors. Instruktoren werden durch die Gruppe der Kursdirektoren dazu ausgewählt, vorgeschlagen und von ihrem NRC oder dem internationalen Kurskomitees ernannt. Kursdirektoren sind erfahrene Persönlichkeiten mit erheblicher klinischer Kompetenz, die ihre Qualitäten als Lehrer und Prüfer unter Beweis gestellt haben. Sie besitzen Führungseigenschaften und haben sich die Unterrichtsprinzipien des Instruktorkurses zu eigen gemacht. Ein Schlüsselelement der ERC-Kurse sind die „faculty meetings" am Anfang und am Ende jedes Kurstages unter Führung des Kursdirektors. Ziel dieser Treffen ist, den Lehrkörper zu instruieren und die Bewertung jedes einzelnen Teilnehmers zu ermöglichen. Am Ende eines Kurses findet das Abschlusstreffen statt, bei dem die Gesamtleistung der einzelnen Teilnehmer beurteilt und über die erfolgreiche Kursteilnahme entschieden wird. Wie oben beschrieben, können Kursteilnehmer mit außergewöhnlicher Leistung zum Instruktortraining eingeladen werden. Die Leistung der IC im Kurs wird ebenfalls beurteilt, und sie erhalten ein Feedback von den Mentoren der Kursdirektoren. Das Faculty meeting gibt auch den Instruktoren die Möglichkeit zum Debriefing des Kurses.

\section{Kurse}

\section{Basic life support und Automated external defibrillation}

BLS/AED-Kurse sind auf weite Teile der Bevölkerung ausgerichtet. Dazu gehören klinische und nichtklinische professionelle Helfer (insbesondere die, die eher selten eine Reanimationssituation bewältigen werden müssen), niedergelassene praktische Ärzte, Zahnärzte, Medizinstudenten, Erste-Hilfe-Personal, Rettungsschwimmer, Personen in Garantenstellung (Lehrer, Sozialarbeiter), am Funktionsplan der innerklinischen Notfallteams Beteiligte und die Allgemeinbevölkerung. Es gibt getrennte
BLS- und AED-Kurse, der ERC empfiehlt allerdings die Kombination.

Provider-Kurs. Das Ziel der ProviderKurse besteht darin, dass jeder Teilnehmer in die Lage versetzt wird, Kompetenzen in BLS-Maßnahmen bzw. in der AED-Anwendung zu erlangen. Jeder BLS/AEDKurs dauert etwa einen halben Tag und beinhaltet vorwiegend die Demonstration der Fertigkeiten und praktischen Übungen, mit wenigen Vorlesungen. Empfohlen wird ein Instruktor-Teilnehmer-Verhältnis von 1:6 mit mindestens einem Übungsphantom und einem AED für jede Gruppe. Offizielle Tests werden normalerweise nicht durchgeführt, jedoch erhält jeder Teilnehmer bei seinen Übungen ein individuelles Feedback. Wer aus beruflichen oder privaten Gründen ein Zeugnis wünscht, wird während des Kurses kontinuierlich oder abschließend am Kursende beurteilt.

Instruktorkurs. Viele der Teilnehmer eines BLS/AED-Provider-Kurses sind Laien; einige wollen anschließend selbst Instruktoren werden. Daher hat der ERC einen eintägigen BLS/AED-Instruktor-Kurs etabliert. Teilnehmer dieses Kurses müssen professionelle Helfer sein oder Laien, die das ERC-BLS/AED-Provider-Zertifikat erworben haben und die Eignung zum Instruktor aufweisen. Das Ziel dieser Instruktorkurse ist, dass alle angehenden Instruktoren durch intensive Kursteilnahme das vorrangige Kriterium erfüllen, selber BLS/ AED-Themen zu unterrichten. Der BLS/ AED-Instruktor-Kurs folgt den Prinzipien des GIC mit dem Schwerpunkt, Laien unterrichten zu können. Nach erfolgreichem Kursabschluss steigen die Teilnehmer zu IC auf und unterrichten 2 BLS/AED-Kurse, bevor sie Full instructor werden.

\section{Immediate life support}

Immediate-life-support-Kurse sind für die Mehrzahl der professionellen Helfer gedacht, die zwar selten mit einem Kreislaufstillstand konfrontiert werden, aber das Potenzial haben, Notfallhelfer oder Mitglieder eines Reanimationsteams zu werden [208]. Der Kurs unterrichtet die Teilnehmer in Fertigkeiten, die Wiederbelebung erfolgreich durchzuführen, während sie auf das Eintreffen des Reanimationsteams warten [209]. Der ILS-Kurs be- 
inhaltet auch Informationen dazu, wie kritisch Kranke zu behandeln sind [210] und wie ein Kreislaufstillstand verhindert werden kann. Eine vor Kurzem veröffentlichte Studie zeigte, dass die Zahl der Reanimationsalarme nach erfolgten Kreislaufstillständen zurückging, während mehr rechtzeitige Alarme vor Kreislaufstillständen abgesetzt wurden, nachdem ein ILS-Kurs in das Trainingsprogramm von 2 Krankenhäusern aufgenommen worden war. Die ILS-Einführung war auch mit der Reduktion realer Kreislaufstillstände, dem Anstieg des primären Überlebens sowie einer höheren Überlebensrate bis zur Entlassung verbunden [211]. Potenzielle Teilnehmer solcher ILS-Kurse sind Krankenpflegepersonal, Krankenpflegeauszubildende, Ärzte, Medizinstudenten, Zahnärzte, Physiotherapeuten, technische Assistenten in der Medizin und Kardiotechniker.

Kursablauf. Der ILS-Kurs ist ein Eintageskurs und beinhaltet Vorträge, praktische Übungen und „Cardiac-arrest“-Simulationszenarien („,cardiac arrest simulation teaching“, CASTeach) am Übungsphantom. Das Programm bietet den Instruktoren die Möglichkeit, den Kurs individuell auf die Teilnehmer auszurichten. Die ILS-Kurse finden im Kleingruppenunterricht (12 Teilnehmer) statt. Es soll die Ausrüstung (z. B. Defibrillator) verwendet werden, mit dem die Teilnehmer im Alltag arbeiten.

Kursinhalt. Der Kurs deckt alle Maßnahmen ab, die wahrscheinlich zu einer erfolgreichen Wiederbelebung führen: Ursache und Vermeidung des Kreislaufstillstands, „Airway-breathing-circulation-disability-exposure“- (ABCDE-)Ansatz, Beginn der Wiederbelebungsmaßnahmen, einfaches Atemwegsmanagement und Defibrillation (mithilfe des AED und manuell). Es besteht die Möglichkeit, relevante Inhalte für spezielle Gruppen (z. B. Anaphylaxie, Check der Ausrüstung) einzubauen. Nachdem alle Techniken gelehrt wurden, führen die Instruktoren die Demonstration eines Kreislaufstillstands durch, die den Teilnehmern ihre spezielle Aufgabe als Notfallhelfer vor Augen führt. Anschließend üben die Teilnehmer an den CASTeach-Stationen praktisch. Normalerweise wird von den ILS-Teilnehmern nicht er- wartet, die Rolle des Teamleiters zu übernehmen. Die Teilnehmer sollen in der Lage sein, Reanimationsversuche zu beginnen und so lange fortzuführen, bis weitere professionelle Hilfe eintrifft. Bei Bedarf übernimmt der Instruktor die Rolle des Teamleiters; dies ist jedoch nicht immer notwendig, da in manchen Szenarien die Reanimation schon vor dem Eintreffen erfahrenerer Helfer erfolgreich ist. Es wird mit standardisierten Szenarien gearbeitet, die an den jeweiligen Arbeitsplatz und die innerklinische Funktion der Teilnehmer angepasst werden.

Bewertung. Die Leistungen der Teilnehmer werden kontinuierlich bewertet; die Teilnehmer müssen während des ganzen Kurses ihr Können zeigen. Es gibt keinen offiziellen Test am Ende des Kurses. Zusammen mit den Vorbereitungsmaterialien erhalten die Teilnehmer die Beurteilungsbögen. Diese Bögen zeigen eindeutig, wie die Leistungen entsprechend der vorgegebenen Kriterien beurteilt werden. Die Bewertungen während des ILS-Kurses erlauben den Teilnehmern zu erkennen, was von ihnen erwartet wird, und richten das Lernen auf diese Ziele aus. Folgende praktische Übungen werden im ILS-Kurs bewertet: Atemwegsmanagement, BLS und Defibrillation. Bei entsprechender Unterstützung erreichen die meisten Teilnehmer das Kursziel.

\section{Advanced life support}

Die Zielgruppe des ALS-Kurses sind Ärzte und erfahrenes Pflegepersonal im Notfallbereich von Krankenhäusern sowie Mitglieder und Leiter von medizinischen Notfall- und Herzalarmteams [212, 213]. Der Kurs ist auch für erfahrenes Rettungsdienst- und spezielles Krankenhauspersonal geeignet. Für Pflegepersonal als Notfallhelfer, für Ärzte, die seltener mit einem Kreislaufstillstand konfrontiert sind, und für Rettungssanitäter ist eher der ILSKurs geeignet. Jeder Instruktor ist Mentor für eine kleine Gruppe von Teilnehmern. Der Kurs dauert normalerweise 2 oder 2,5 Tage.

Kursablauf. Der Kurs beinhalte wenige reguläre Vorlesungen; der Unterricht konzentriert sich auf praktische Übungen, klinisch basierte Szenarien in Kleingruppen mit dem Schwerpunkt des Teamleiteransatzes und interaktiven Gruppendiskussionen. Eine offizielle Mentorensitzung dient dem Feedback der Teilnehmer.

Kursinhalt. Der Kursinhalt basiert auf den gültigen ERC-Leitlinien für Wiederbelebung. Von den Teilnehmern wird erwartet, dass sie das ALS-Kurs-Manual bereits vor dem Kurs sorgfältig durchgearbeitet haben. Kursziele für die Teilnehmer sind: Ursachen eines Kreislaufstillstands erkennen zu können, kritisch kranke Menschen, bei denen die Gefahr besteht, dass sich ihr Zustand verschlechtern wird, identifizieren zu können, den Patienten mit Kreislaufstillstand adäquat versorgen zu können und Probleme im $\mathrm{Zu}$ sammenhang mit der Peri-arrest-Situation, die innerhalb der ersten Stunde nach Eintritt des Kreislaufstillstands auftreten, meistern zu können. Es ist kein Kurs in erweiterter Intensivbehandlung oder in Kardiologie. Die Beherrschung der BLSMaßnahmen wird vorausgesetzt, wenn sich Teilnehmer für den Kurs anmelden.

Kursschwerpunkte liegen in der Technik der sicheren Defibrillation und der EKG-Interpretation, dem Atemwegs- und Beatmungsmanagement, der Behandlung von Peri-arrest-Arrhythmien, der Korrektur des Säure-Basen-Haushalts und im Management spezieller Reanimationssituationen. Reanimationsnachsorge, ethische Aspekte im Zusammenhang mit der Reanimation und die Betreuung von Angehörigen sind ebenfalls Inhalt des Kurses.

Beurteilung und Test. Jeder Teilnehmer wird während des Kurses kontinuierlich beurteilt und am Ende jedes Tages im Faculty meeting bewertet. Von den Teilnehmern wird während des Kursverlaufs die Anwendung des ABCDE-Ansatzes zur Beurteilung von kritisch Kranken erwartet, das Erkennen eines Kreislaufstillstands, die qualitativ hochwertige CPR und die sichere Defibrillation. Am Ende des Kurses findet ein Simulationstest („cardiac arrest simulation testing“, CASTest) statt, der überprüft, wie die Teilnehmer ihr Wissen und ihre Fertigkeiten während eines simulierten Kreislaufstillstands anwenden. Reliabilität und Aussagekraft des CASTests wurden publiziert $[125,169,214]$. Mithilfe eines Multi- 
ple-Choice-Verfahrens wird am Kursende das Wissen überprüft; die Teilnehmer müssen $75 \%$ der Fragen richtig beantworten, um den Kurs erfolgreich abzuschließen. Dieser „Multiple-choice-question“(MCQ-)Test zeigte bei einer Evaluation von über 8000 Kursteilnehmern hohe interne Konsistenz- und Diskriminierungseigenschaften (Daten des Resuscitation Council UK und Dr. Carl Gwinnutt).

\section{European paediatric life support}

Der EPLS-Kurs ist für Mitarbeiter im Gesundheitswesen gedacht, die prä- oder innerklinisch in die Reanimation von Neugeborenen, Säuglingen oder Kindern involviert sind. Der Kurs zielt darauf ab, die Teilnehmer mit Wissen und Fertigkeiten auszustatten, um ein kritisch krankes Kind während der ersten Stunde zu versorgen und eine Verschlechterung des $\mathrm{Zu}$ stands bis hin zum Kreislaufstillstand zu verhindern.

Dies ist kein Kurs in Neugeborenenoder Kinderintensivmedizin für Fortgeschrittene. Das Beherrschen der Basismaßnahmen im Paediatric life support ist Voraussetzung, obwohl eine Auffrischung in BLS und eine Unterweisung in Maßnahmen bei Verlegung der Atemwege durch Fremdkörper zum Kurs gehören. Der EPLS-Kurs ist geeignet für Ärzte, Pflegepersonal, Rettungssanitäter und assistenten etc., die mit der Versorgung von kranken Neugeborenen, Säuglingen und Kindern betraut sind [216, 217]. Um die Szenarien realistisch auszurichten und um die Fragen der Teilnehmer beantworten zu können, müssen 50\% der Instruktoren regelmäßige Erfahrung in der Behandlung von Neugeborenen und Kindern haben. Der Kurs dauert 2 bis 2,5 Tage.

Kursablauf. Das Kurskonzept enthält wenige Vorlesungen. Kenntnisse und Fertigkeiten werden in Kleingruppen anhand klinikbasierter Szenarien vermittelt (z. B. Kreislaufstillstand, Atemversagen, Simulation der Behandlung nach einer Geburt). Der Schwerpunkt liegt auf der Untersuchung und der Behandlung des kranken Kindes, auf Teamarbeit und Führungsverhalten.

Kursinhalt. Der Kursinhalt basiert auf den gültigen ERC-Leitlinien zur Reani- mation von Neugeborenen und Kindern. Von den Teilnehmern wird erwartet, dass sie das Kursmanual bereits vor dem Kurs sorgfältig durchgearbeitet haben. Rund 4 bis 6 Wochen vor dem Kurs erhalten die Teilnehmer zusammen mit dem Manual einen Eingangstest, um eine bessere Vorbereitung zu ermöglichen. Ziel des EPLSKurs ist es, die Teilnehmer in den Ursachen und Mechanismen eines Atem-/ Kreislaufstillstands beim Neugeborenen und Kind auszubilden, das kritisch kranke Neugeborene, den kritisch kranken Säugling oder das kritisch kranke Kind $\mathrm{zu}$ erkennen sowie $\mathrm{zu}$ behandeln und wenn nötig Reanimationsmaßnahmen durchführen zu können. Folgende Fertigkeiten werden unterrichtet: Atemwegsmanagement, Beutel-Maske-Beatmung, achsengerechte Drehung und Anlegen einer Hals-Wirbel-Säulen- (HWS-)Stabilisierung, Verwendung von Sauerstoff, Einführung in die endotracheale Intubation und die Anlage eines intravenösen $\mathrm{Zu-}$ gangs, sichere Defibrillation, Kardioversion sowie die Anwendung eines AED.

Jeder Teilnehmer wird vom Instruktorenteam individuell bewertet und beurteilt. Wenn notwendig, wird ein Feedback gegeben. Nach der BLS-Auffrischung werden die BLS-Fertigkeiten bewertet; am Ende des Kurses legt die Beurteilung eines szenarienbasierten Tests den Schwerpunkt auf die Untersuchung des kranken Kindes und andere zentrale Fertigkeiten. Mithilfe eines schriftlichen Multiple-Choice-Tests mit einer Bestehensquote von $75 \%$ wird am Ende des Kurses das theoretische Wissen überprüft.

\section{European pediatric immediate life support}

Kursablauf. Der EPILS-Kurs findet an einem einzelnen Tag statt, mit einem Vortrag, praktischen Übungen und simulierten Szenarien. Es besteht die Möglichkeit, den Kurs an die individuellen Bedürfnisse der Teilnehmer auszurichten.

Kursinhalt. Das Ziel des Kurses ist, Pflegedienstpersonal, Rettungssanitäter und Ärzten das Erkennen und Behandeln kritische kranker Neugeborener, Säuglinge oder Kinder zu vermitteln, einem Kreislaufstillstand vorzubeugen und Kinder im
Kreislaufstillstand während der ersten Minuten und des Wartens auf ein Reanimationsteam zu versorgen.

Dieser interaktive Kurs basiert auf kurzen praktischen Simulationen, die an den Arbeitsplatz und die aktuelle Rolle der Teilnehmer angepasst sind.

Basic Life Support, Beutel-Maske-Beatmung, Thoraxkompression, die Behandlung des Beinaheerstickens und das Legen introssärer Zugänge werden unterrichtet; Medikamente während eines Kreislaufstillstands und das Einlegen einer Larynxmaske sind optionale Kursinhalte.

Der EPILS-Kurs ist auf einfaches Ausrichten konzipiert. Die meisten Kurse finden in Kliniken mit kleinen Gruppen (5 bis 6 Teilnehmer mit einem Instruktor) statt. Zumindest ein Baby- und ein Kinderübungsphantom für je 6 Teilnehmer sind erforderlich. Die Kurszentren sollen nach Möglichkeit mit der Ausrüstung trainieren, mit der im klinischen Alltag gearbeitet wird (Defibrillator).

Bewertung. Jedem Teilnehmer wird vor dem Kurs mit dem Kursmanual ein Eingangstest, der zur besseren Vorbereitung dienen soll, zugeschickt. Diese Vorbereitung fließt nicht in die Abschlussbeurteilung ein. Während des Kurses gibt es keinen offiziellen Test, obwohl die Teilnehmer kontinuierlich auf ihre Fortschritte hin überprüft werden. $\mathrm{Zu}$ Beginn des Kurses werden den Teilnehmern Bewertungsformulare ausgehändigt, und während des Kurses erhalten sie regelmäßiges Feedback von den Instruktoren.

Die folgenden praktischen Fertigkeiten werden überprüft: Basismaßnahmen der Reanimation, Beutel-Maske-Beatmung und der Gebrauch eines AED. Mit der unterstützenden Einstellung der Instruktoren erreichen die meisten Kursteilnehmer die Kursziele.

\section{Newborn life support}

Der Eintages-NLS-Kurs ist auf Mitarbeiter im Gesundheitswesen ausgerichtet, die berufsbedingt bei einer Geburt eines Kindes anwesend sein können. Ziel ist es, jedem, der zu einer Geburt hinzugerufen werden kann, das nötige Hintergrundwissen und die Fertigkeiten zu vermitteln, um innerhalb der ersten 10-20 min effektive Reani- 
mationsmaßnahmen durchzuführen. Der Kurs ist auf Hebammen, Pflegepersonal, Rettungssanitäter und Ärzte zugeschnitten und gelingt - wie die meisten derartigen Kurse - am besten mit Teilnehmern aus verschiedenen Professionen.

Kursablauf. Das NLS-Manual wird dem Teilnehmer 4 Wochen vor Kursbeginn zugesendet. Jeder Teilnehmer erhält mit dem Manual einen Multiple-Choice-Fragebogen und wird ersucht, diesen ausgefüllt zum Kurs mitzubringen. Nach der Kurseinleitung werden 2 Referate gehalten, danach werden die Teilnehmer in 4 Gruppen eingeteilt und durchlaufen bereits vor der Mittagspause 3 Übungsstationen. Der Nachmittag beginnt mit einer Szenariodemonstration, gefolgt von 2-stündigem Simulationsunterricht in Kleingruppen. Abschließend finden eine theoretische und eine praktische Überprüfung mit einem Multiple-ChoiceTest und einem praktischen individuellen Atemwegsmanagementtest statt. Der Kurs konzentriert sich auf das Atemwegsmanagement, behandelt aber auch Thoraxkompression, Nabelvenenzugang und Medikamente.

Neugeborenenübungsphantome für BLS-, aber auch 4 Übungsphantome für ALS-Maßnahmen sowie die Trainingsausrüstung für das Atemwegsmanagement sollen verfügbar sein. Neugeborenenbehandlungsplätze, idealerweise mit ausreichender Gasversorgung für den Tag, werden benötigt.

\section{Generic instructor course}

Dieser Kurs ist für IP aus einem ERC-Kurs (ALS, EPLS, NLS, ILS, EPILS) gedacht. Teilnehmer mit dem IP-Status eines anderen Kursformats [z. B. „European trauma course", prähospitale Traumaversorgung (Italien)], können den GIC ebenfalls belegen. Am Kurs nehmen maximal 24 Teilnehmer (in 4 Gruppen) bei einem Instruktor-Teilnehmer-Verhältnis von 1:3 teil. Alle Instruktoren müssen erfahrene ERC-Instruktoren sein, die durch eine offizielle Weiterbildung den GIC-Instruktor-Status erlangt haben. Das Hauptaugenmerk dieses Kurses liegt auf der Vermittlung von Ausbildungs- und Prüfungsfertigkeiten, dem Fördern von Führungsqualitäten und dem Vermitteln des kons- truktiven Feedbacks. Die Kenntnisse der Provider-Kurse werden beim GIC vorausgesetzt. Der Kurs dauert 2 oder 2,5 Tage.

Kursablauf. Der GIC ist ein überwiegend interaktiver Kurs. Ein ERC-Educator übernimmt die Schlüsselrolle in der Leitung des Lehrprozesses, in den Diskussionen und beim Feedback. Vorlesungen sind mit Gruppenaktivitäten verbunden wie Kleingruppendiskussionen, Üben von didaktischen Fertigkeiten und Simulationseinheiten. Es finden Mentorensitzungen sowie zum Beginn und am Ende der Kurstage ein Faculty meeting statt.

Kursinhalt. Von den Teilnehmern wird erwartet, dass sie bereits vor dem Kurs das GIC-Manual durchgearbeitet haben. $\mathrm{Zu}$ Beginn des Kurses werden der theoretische Hintergrund der Erwachsenenbildung und die Grundlagen effektiven Lehrens und Prüfens vom Educator vorgestellt. Jede Unterrichts- und Beurteilungsmethode wird von den Instruktoren demonstriert. Danach haben die Teilnehmer die Möglichkeit zu praktizieren: Routineerwerb im Gebrauch der Ausrüstung, Halten von Vorlesungen, Lehren von Fertigkeiten mithilfe des „4-step approach“, einfache Simulation, Kleingruppenunterricht (offene und geschlossene Diskussion) sowie Beurteilung. Unterrichtsmaterial für jede Unterrichtseinheit sind kurze Abschnitte aus den Original-ERC-Kursen. Der Schwerpunkt während des Kurses liegt in der Rolle des Instruktors. Jeder Teilnehmer hat die Möglichkeit, in die Rolle des Instruktors hineinzuwachsen. Konstruktives Feedback ist ein Schlüsselelement des Kurses. Abschließend werden die Rollen und Qualitäten eines Instruktors diskutiert.

Bewertung. Jeder Teilnehmer steht unter kontinuierlicher Bewertung durch das Instruktorenteam. Leistung und Einstellung der Teilnehmer werden während der Besprechungen der Instruktoren diskutiert, und bei Bedarf wird ein Feedback gegeben. Erfolgreiche Teilnehmer erhalten den Status des IC. Erfolgreichen GIC-Teilnehmern, die noch weitere Unterstützung in ihrer Entwicklung zum IC brauchen, wird vom Lehrkörper empfohlen, in zugewie- senen Zentren ihr Wissen und ihre Fertigkeiten weiter zu ergänzen.

\section{Educator master class}

Medizinische Lehrer („medical educator") sind ein essenzieller Bestanteil der GIC-Faculty. Dieser Zweitageskurs ist für erfahrene Trainer, die ERC Medical educator werden wollen, und wird vom ERC je nach Bedarf an Medical educators abgehalten. Geeignete Kandidaten werden von der ERC Educational Advisory Group (ERC-EAG) nach schriftlicher Bewerbung ausgewählt. Entsprechender Hintergrund und Lehrerfahrung sowie eine Qualifikation in medizinischer Lehre oder spezielles Engagement in der Aus- und Weiterbildung über Jahre sind weitere Voraussetzungen. Erfahrung aus ERC-Kursen und dem GIC sowie das Lesen der Hintergrundliteratur für den Kurs werden von den Teilnehmern erwartet. Die Instruktoren dieses Kurses sind erfahrene Educators.

Kursablauf. Der Kurs besteht vorwiegend aus geschlossenen Diskussionen, die jeweils von 1 bis 2 Educators geleitet werden. Dies wird durch Kleingruppendiskussionen und Problemlösestrategien in Kleingruppen ergänzt.

Kursinhalt. Der Kurs umfasst den theoretischen Rahmen für die medizinischen Educators, Bewertung und Qualitätskontrolle, Unterrichtsmethoden, kritische Beurteilung, die Rolle des Mentors, multiprofessionelle Lehrstrategien und die kontinuierliche Entwicklung des medizinischen Educator.

Bewertung. Während des gesamten Kurses steht jeder Teilnehmer unter offizieller Bewertung durch den Lehrkörper. Erfolgreiche Teilnehmer erhalten den Status eines "educator candidate“ (EC), die dann durch erfahrene Educators und GICKursdirektoren so lange supervidiert und bewertet werden, bis entschieden wird, ob sie als selbstständige Educators eingesetzt werden oder nicht. 
Korrespondierender Übersetzer

Prof. Dr. med. Robert Greif, MME

Universitätsklinik für Anästhesiologie

und Schmerztherapie

Universitätshospital Bern

Inselspital, 3010 Bern

Schweiz

Robert.Greif@insel.ch

Interessenkonflikt. Der korrespondierende Übersetzer gibt an, dass kein Interessenkonflikt besteht.

\section{Literatur}

1. Chamberlain DA, Hazinski MF (2003) Education in resuscitation. Resuscitation 59:11-43

2. Yeung J, Perkins GD (2010) Timing of drug administration during CPR and the role of imulation. Resuscitation 81:265-266

3. Berdowski J, Schmohl A, Tijssen JG, Koster RW (2009) Time needed for a regional emergency medical system to implement resuscitation Guidelines 2005-The Netherlands experience. Resuscitation 80:1336-1341

4. Bigham BL, Koprowicz K, Aufderheide TP et al (2010) Delayed Prehospital Implementation of the 2005 American Heart Association Guidelines for Cardiopulmonary Resuscitation and Emergency Cardiac Care. Prehosp Emerg Care

5. Soar J, Mancini ME, Bhanji F et al (In Press) 2010 International Consensus on Cardiopulmonary Resuscitation and Emergency Cardiovascular Care Science with Treatment recommendations. Part 12: Education, Implementation, and Teams. Resuscitation

6. Baskett PJ, Nolan JP, Handley A et al (2005) European Resuscitation Council guidelines for resuscitation 2005. Section 9. Principles of training in resuscitation. Resuscitation 67(Suppl 1):S181-S189

7. Andersen PO, Jensen MK, Lippert A, Ostergaard D (2010) Identifying non-technical skills and barriers for improvement of teamwork in cardiac arrest teams. Resuscitation 81:695-702

8. Flin R, Patey R, Glavin R, Maran N (2010) Anaesthetists' non-technical skills. Br J Anaesth

9. Axelsson A, Thoren A, Holmberg S, Herlitz J (2000) Attitudes of trained Swedish lay rescuers toward CPR performance in an emergency: a survey of 1012 recently trained CPR rescuers. Resuscitation 44:27-36

10. Hubble MW, Bachman M, Price R et al (2003) Willingness of high school students to perform cardiopulmonary resuscitation and automated external defibrillation. Prehosp Emerg Care 7:219-224

11. Swor RA, Jackson RE, Compton S et al (2003) Cardiac arrest in private locations: different strategies are needed to improve outcome. Resuscitation 58:171-176

12. Swor R, Khan I, Domeier R et al (2006) CPR training and CPR performance: do CPR-trained bystanders perform CPR? Acad Emerg Med 13:596601

13. Vaillancourt C, Stiell IG, Wells GA (2008) Understanding and improving low bystander CPR rates: a systematic review of the literature. CJEM 10:5165
14. Boucek CD, Phrampus P, Lutz J et al (2009) Willingness to perform mouth-tomouth ventilation by health care providers: a survey. Resuscitation 80:849-853

15. Caves ND, Irwin MG (2006) Attitudes to basic life support among medical students following the 2003 SARS outbreak in Hong Kong. Resuscitation 68:93-100

16. Coons SJ, Guy MC (2009) Performing bystander CPR for sudden cardiac arrest: behavioural intentions among the general adult population in Arizona. Resuscitation 80:334-340

17. Dwyer T (2008) Psychological factors inhibit family members' confidence to initiate CPR. Prehosp Emerg Care 12:157-161

18. Jelinek GA, Gennat H, Celenza T et al (2001) Community attitudes towards performing cardiopulmonary resuscitation in Western Australia. Resuscitation 51:239-246

19. Johnston TC, Clark MJ, Dingle GA, FitzGerald G (2003) Factors influencing Queenslanders' willingness to perform bystander cardiopulmonary resuscitation. Resuscitation 56:67-75

20. Kuramoto N, Morimoto T, Kubota Y et al (2008) Public perception of and willingness to perform bystander CPR in Japan. Resuscitation 79:475481

21. Omi W, Taniguchi T, Kaburaki T et al (2008) The attitudes of Japanese high school students toward cardiopulmonary resuscitation. Resuscitation 78:340-345

22. Riegel B, Mosesso VN, Birnbaum A et al (2006) Stress reactions and perceived difficulties of lay responders to a medical emergency. Resuscitation 70:98-106

23. Shibata K, Taniguchi T, Yoshida M, Yamamoto K (2000) Obstacles to bystander cardiopulmonary resuscitation in Japan. Resuscitation 44:187-193

24. Taniguchi T, Omi W, Inaba H (2007) Attitudes toward the performance of bystander cardiopulmonary resuscitation in Japan. Resuscitation 75:82-87

25. Moser DK, Dracup K, Doering LV (1999) Effect of cardiopulmonary resuscitation training for parents of high-risk neonates on perceived anxiety, control, and burden. Heart Lung 28:326-333

26. Axelsson A, Herlitz J, Ekstrom L, Holmberg S (1996) Bystander-initiated cardiopulmonary resuscitation out-of-hospital. A first description of the bystanders and their experiences. Resuscitation 33:3-11

27. Donohoe RT, Haefeli K, Moore F (2006) Public perceptions and experiences of myocardial infarction, cardiac arrest and CPR in London. Resuscitation 71:70-79

28. Hamasu S, Morimoto T, Kuramoto N et al (2009) Effects of BLS training on factors associated with attitude toward CPR in college students. Resuscitation 80:359-364

29. Parnell MM, Pearson J, Galletly DC, Larsen PD (2006) Knowledge of and attitudes towards resuscitation in New Zealand high-school students. Emerg Med J 23:899-902

30. Swor R, Compton S, Farr L et al (2003) Perceived self-efficacy in performing and willingness to learn cardiopulmonary resuscitation in an elderly population in a suburban community. Am J Crit Care 12:65-70
31. Koster RW, Baubin MA, Caballero A et al (2010) European Resuscitation Council Guidelines for Resuscitation 2010. Section 2. Adult basic life support and use of automated external defibrillators. Resuscitation 81

32. Koster RW, Sayre MR, Botha M et al (In Press) 2010 International Consensus on Cardiopulmonary Resuscitation and Emergency Cardiovascular Care Science with Treatment ecommendations. Part 5: Adult Basic Life Support. Resuscitation

33. Perkins GD, Walker G, Christensen K et al (2006) Teaching recognition of agonal breathing improves accuracy of diagnosing cardiac arrest. Resuscitation 70:432-437

34. Bobrow BJ, Zuercher M, Ewy GA et al (2008) Gasping during cardiac arrest in humans is frequent and associated with improved survival. Circulation 118:2550-2554

35. Yeung J, Meeks R, Edelson D et al (2009) The use of CPR feedback/ prompt devices during training and CPR performance: A systematic review. Resuscitation 80:743-751

36. Lam KK, Lau FL, Chan WK, Wong WN (2007) Effect of severe acute respiratory syndrome on bystander willingness to perform cardiopulmonary resuscitation (CPR) - is compression-only preferred to standard CPR? Prehosp Disaster Med 22:325329

37. Locke CJ, Berg RA, Sanders AB et al (1995) Bystander cardiopulmonary resuscitation. Concerns about mouth-to-mouth contact. Arch Intern Med 155:938-943

38. Kitamura T, Iwami T, Kawamura T et al (2010) Conventional and chest-compression-only cardiopulmonary resuscitation by bystanders for children who have out-of-hospital cardiac arrests: a prospective, nationwide, population-based cohort study. Lancet

39. Biarent D, Bingham R, Eich C et al (2010) European Resuscitation Council Guidelines for Resuscitation 2010. Section 6. Paediatric Life Support. Resuscitation 81

40. Hoke RS, Chamberlain DA, Handley AJ (2006) A reference automated external defibrillator provider course for Europe. Resuscitation 69:421-433

41. Lynch B, Einspruch EL, Nichol G et al (2005) Effectiveness of a 30-min CPR self-instruction program for lay responders: a controlled randomized study. Resuscitation 67:31-43

42. Todd KH, Braslow A, Brennan RT et al (1998) Randomized, controlled trial of video self-instruction versus traditional CPR training. Ann Emerg Med 31:364-369

43. Einspruch EL, Lynch B, Aufderheide TP et al (2007) Retention of CPR skills learned in a traditional AHA Heartsaver course versus 30 -min video selftraining: a controlled randomized study. Resuscitation 74:476-486

44. Todd KH, Heron SL, Thompson M et al (1999) Simple CPR: a randomized, controlled trial of video self-instructional cardiopulmonary resuscitation training in an African American church congregation. Ann Emerg Med 34:730-737

45. Reder S, Cummings P, Quan L (2006) Comparison of three instructional methods for teaching cardiopulmonary resuscitation and use of an automatic external defibrillator to high school students. Resuscitation 69:443-453 
46. Roppolo LP, Pepe PE, Campbell L et al (2007) Prospective, randomized trial of the effectiveness and retention of 30-min layperson training for cardiopulmonary resuscitation and automated external defibrillators: The American Airlines Study. Resuscitation 74:276-285

47. Batcheller AM, Brennan RT, Braslow A et al (2000) Cardiopulmonary resuscitation performance of subjects over forty is better following halfhour video self-instruction compared to traditional four-hour classroom training. Resuscitation 43:101-110

48. Braslow A, Brennan RT, Newman MM et al (1997) CPR training without an instructor: development and evaluation of a video self-instructional system for effective performance of cardiopulmonary resuscitation. Resuscitation 34:207-220

49. Isbye DL, Rasmussen LS, Lippert FK et al (2006) Laypersons may learn basic life support in $24 \mathrm{~min}$ using a personal resuscitation manikin. Resuscitation 69:435-442

50. Moule P, Albarran JW, Bessant E et al (2008) A non-randomized comparison of e-learning and classroom delivery of basic life support with automated external defibrillator use: a pilot study. Int J Nurs Pract 14:427-434

51. Liberman M, Golberg N, Mulder D, Sampalis J (2000) Teaching cardiopulmonary resuscitation to CEGEP students in Quebec - a pilot project. Resuscitation 47:249-257

52. Jones I, Handley AJ, Whitfield R et al (2007) A preliminary feasibility study of a short DVD-based distance-learning package for basic life support. Resuscitation 75:350-356

53. Brannon TS, White LA, Kilcrease JN et al (2009) Use of instructional video to prepare parents for learning infant cardiopulmonary resuscitation. Proc (Bayl Univ Med Cent) 22:133-137

54. Vries W de, Turner N, Monsieurs K et al (In Press) Comparison of instructor-led Automated External Defibrillation training and three alternative DVDbased training methods. Resuscitation

55. Perkins GD, Mancini ME (2009) Resuscitation training for healthcare workers. Resuscitation 80:841-842

56. Mattei LC, McKay U, Lepper MW, Soar J (2002) Do nurses and physiotherapists require training to use an automated external defibrillator? Resuscitation 53:277-280

57. Gundry JW, Comess KA, DeRook FA et al (1999) Comparison of naive sixthgrade children with trained professionals in the use of an automated external defibrillator. Circulation 100:1703-1707

58. Beckers S, Fries M, Bickenbach J et al (2005) Minimal instructions improve the performance of laypersons in the use of semiautomatic and automatic external defibrillators. Crit Care 9:R110-R116

59. Beckers SK, Fries M, Bickenbach J et al (2007) Retention of skills in medical students following minimal theoretical instructions on semi and fully automated external defibrillators. Resuscitation 72:444-450

60. Mitchell KB, Gugerty L, Muth E (2008) Effects of brief training on use of automated external defibrillators by people without medical expertise. Hum Factors 50:301-310

61. Jerin JM, Ansell BA, Larsen MP, Cummins RO (1998) Automated external defibrillators: skill maintenance using computer-assisted learning. Acad Emerg Med 5:709-717
62. Vries W de, Handley AJ (2007) A web-based micro-simulation program for self-learning BLS skills and the use of an AED. Can laypeople train themselves without a manikin? Resuscitation 75:491-498

63. Spooner BB, Fallaha JF, Kocierz L et al (2007) An evaluation of objective feedback in basic life support (BLS) training. Resuscitation 73:417-424

64. Andresen D, Arntz HR, Grafling W et al (2008) Public access resuscitation program including defibrillator training for laypersons: a randomized trial to evaluate the impact of training course duration. Resuscitation 76:419-424

65. Smith KK, Gilcreast D, Pierce K (2008) Evaluation of staff's retention of ACLS and BLS skills. Resuscitation 78:59-65

66. Woollard M, Whitfeild R, Smith A et al (2004) Skill acquisition and retention in automated external defibrillator (AED) use and CPR by lay responders: a prospective study. Resuscitation 60:17-28

67. Berden HJ, Willems FF, Hendrick JM et al (1993) How frequently should basic cardiopulmonary resuscitation training be repeated to maintain adequate skills? BMJ 306:1576-1577

68. Woollard M, Whitfield R, Newcombe RG et al (2006) Optimal refresher training intervals for AED and CPR skills: a randomised controlled trial. Resuscitation 71:237-247

69. Riegel B, Nafziger SD, McBurnie MA et al (2006) How well are cardiopulmonary resuscitation and automated external defibrillator skills retained over time? Results from the Public Access Defibrillation (PAD) Trial. Acad Emerg Med 13:254-263

70. Castle N, Garton H, Kenward G (2007) Confidence vs competence: basic life support skills of health professionals. Br J Nurs 16:664-666

71. Wik L, Myklebust $H$, Auestad BH, Steen PA (2005) Twelve-month retention of CPR skills with automatic correcting verbal feedback. Resuscitation 66:27-30

72. Christenson J, Nafziger S, Compton S et al (2007) The effect of time on CPR and automated external defibrillator skills in the Public Access Defibrillation Trial. Resuscitation 74:52-62

73. Niles D, Sutton RM, Donoghue A et al (2009) Rolling Refreshers: a novel approach to maintain CPR psychomotor skill competence. Resuscitation 80:909-912

74. Beckers SK, Skorning MH, Fries M et al (2007) CPREzy improves performance of external chest compressions in simulated cardiac arrest. Resuscitation 72:100-107

75. Isbye DL, Hoiby P, Rasmussen MB et al (2008) Voice advisory manikin versus instructor facilitated training in cardiopulmonary resuscitation. Resuscitation 79:73-81

76. Monsieurs KG, De Regge M, Vogels C, Calle PA (2005) Improved basic life support performance by ward nurses using the CAREvent Public Access Resuscitator (PAR) in a simulated setting. Resuscitation 67:45-50

77. Sutton RM, Donoghue A, Myklebust H et al (2007) The voice advisory manikin (VAM): an innovative approach to pediatric lay provider basic life support skill education. Resuscitation 75:161-168

78. Wik L, Myklebust H, Auestad BH, Steen PA (2002) Retention of basic life support skills 6 months after training with an automated voice advisory manikin system without instructor involvement. Resuscitation 52:273-279
79. Nishisaki A, Nysaether J, Sutton R et al (2009) Effect of mattress deflection on CPR quality assessment for older children and adolescents. Resuscitation 80:540-545

80. Perkins GD, Kocierz L, Smith SC et al (2009) Compression feedback devices over estimate chest compression depth when performed on a bed. Resuscitation 80:79-82

81. Perkins GD, Boyle W, Bridgestock H et al (2008) Quality of CPR during advanced resuscitation training. Resuscitation 77:69-74

82. Jensen ML, Lippert F, Hesselfeldt R et al (2009) The significance of clinical experience on learning outcome from resuscitation training-a randomised controlled study. Resuscitation 80:238-243

83. Ali J, Howard M, Williams J (2002) Is attrition of advanced trauma life support acquired skills affected by trauma patient volume? Am J Surg 183:142-145

84. Thomas EJ, Taggart B, Crandell S et al (2007) Teaching teamwork during the Neonatal Resuscitation Program: a randomized trial. J Perinatol 27:409-414

85. Cooper S (2001) Developing leaders for advanced life support: evaluation of a training programme. Resuscitation 49:33-38

86. Gilfoyle E, Gottesman R, Razack S (2007) Development of a leadership skills workshop in paediatric advanced resuscitation. Med Teach 29:e276-e283

87. DeVita MA, Schaefer J, Lutz J et al (2005) Improving medical emergency team (MET) performance using a novel curriculum and a computerized human patient simulator. Qual Saf Health Care 14:326-331

88. Cooper S, Wakelam A (1999) Leadership of resuscitation teams: „Lighthouse Leadership'. Resuscitation 42:27-45

89. Edelson DP, Litzinger B, Arora V et al (2008) Improving in-hospital cardiac arrest process and outcomes with performance debriefing. Arch Intern Med 168:1063-1069

90. Hayes CW, Rhee A, Detsky ME et al (2007) Residents feel unprepared and unsupervised as leaders of cardiac arrest teams in teaching hospitals: a survey of internal medicine residents. Crit Care Med 35:1668-1672

91. Hunziker S, Tschan F, Semmer NK et al (2009) Hands-on time during cardiopulmonary resuscitation is affected by the process of teambuilding: a prospective randomised simulator-based trial. BMC Emerg Med 9:3

92. Makinen M, Aune S, Niemi-Murola L et al (2007) Assessment of CPR-D skills of nurses in Goteborg, Sweden and Espoo, Finland: teaching leadership makes a difference. Resuscitation 72:264-269

93. Marsch SC, Muller C, Marquardt K et al (2004) Human factors affect the quality of cardiopulmonary resuscitation in simulated cardiac arrests. Resuscitation 60:51-56

94. Morey JC, Simon R, Jay GD et al (2002) Error reduction and performance improvement in the emergency department through formal teamwork training: evaluation results of the MedTeams project. Health Serv Res 37:15531581

95. Perkins GD, Davies RP, Soar J, Thickett DR (2007) The impact of manual defibrillation technique on no-flow time during simulated cardiopulmonary resuscitation. Resuscitation 73:109-114 
96. Perkins GD, Lockey AS (2008) Defibrillation-Safety versus efficacy. Resuscitation 79:1-3

97. Perkins GD, Barrett $H$, Bullock I et al (2005) The Acute Care Undergraduate TEaching (ACUTE) Initiative: consensus development of core competencies in acute care for undergraduates in the United Kingdom. Intensive Care Med 31:16271633

98. DeVita MA, Smith GB, Adam SK et al (2010) Identifying the hospitalised patient in crisis - a consensus conference on the afferent limb of rapid response systems. Resuscitation 81:375-382

99. Schwid HA, Rooke GA, Ross BK, Sivarajan M (1999) Use of a computerized advanced cardiac life support simulator improves retention of advanced cardiac life support guidelines better than a textbook review. Crit Care Med 27:821-824

100. Polglase RF, Parish DC, Buckley RL et al (1989) Problem-based ACLS instruction: A model approach for undergraduate emergency medical education. Ann Emerg Med 18:997-1000

101. Clark LJ, Watson J, Cobbe SM et al (2000) CPR,98: a practical multimedia computer-based guide to cardiopulmonary resuscitation for medical students. Resuscitation 44:109-117

102. Hudson JN (2004) Computer-aided learning in the real world of medical education: does the quality of interaction with the computer affect student learning? Med Educ 38:887-895

103. Jang KS, Hwang SY, Park SJ et al (2005) Effects of a Web-based teaching method on undergraduate nursing students' learning of electrocardiography. J Nurs Educ 44:35-39

104. Kim JH, Kim WO, Min KT et al (2002) Learning by computer simulation does not lead to better test performance than textbook study in the diagnosis and treatment of dysrhythmias. J Clin Anesth 14:395-400

105. Leong SL, Baldwin CD, Adelman AM (2003) Integrating Web-based computer cases into a required clerkship: development and evaluation. Acad Med 78:295-301

106. Rosser JC, Herman B, Risucci DA et al (2000) Effectiveness of a CD-ROM multimedia tutorial in transferring cognitive knowledge essential for laparoscopic skill training. Am J Surg 179:320-324

107. Papadimitriou L, Xanthos T, Bassiakou E et al (2010) Distribution of pre-course BLS/AED manuals does not influence skill acquisition and retention in lay rescuers: a randomised study. Resuscitation 81:348-352

108. Perkins GD, Fullerton JN, Davis-Gomez $N$ et al (2010) The effect of pre-course e-learning prior to advanced life support training: A randomised controlled trial. Resuscitation

109. Gerard JM, Scalzo AJ, Laffey SP et al (2006) Evaluation of a novel Webbased pediatric advanced life support course. Arch Pediatr Adolesc Med 160:649-655

110. Xie ZZ, Chen JJ, Scamell RW, Gonzalez MA (1999) An interactive multimedia training system for advanced cardiac life support. Comput Methods Programs Biomed 60:117-131

111. Buzzell PR, Chamberlain VM, Pintauro SJ (2002) The effectiveness of web-based, multimedia tutorials for teaching methods of human body composition analysis. Adv Physiol Educ 26:21-29
112. Christenson J, Parrish K, Barabe S et al (1998) A comparison of multimedia and standard advanced cardiac life support learning. Acad Emerg Med 5:702-708

113. Engum SA, Jeffries $P$, Fisher $L$ (2003) Intravenous catheter training system: computer-based education versus traditional learning methods. Am J Surg 186:67-74

114. Flynn ER, Wolf ZR, McGoldrick TB et al (1996) Effect of three teaching methods on a nursing staff's knowledge of medication error risk reduction strategies. J Nurs Staff Dev 12:19-26

115. Fordis M, King JE, Ballantyne CM et al (2005) Comparison of the instructional efficacy of Internetbased CME with live interactive CME workshops: a randomized controlled trial. JAMA 294:10431051

116. Goldrick B, Appling-Stevens S, Larson E (1990) Infection control programmed instruction: an alternative to classroom instruction in baccalaureate nursing education. J Nurs Educ 29:20-25

117. Harrington SS, Walker BL (2002) A comparison of computer-based and instructor-led training for long-term care staff. J Contin Educ Nurs 33:39-45

118. Jeffries PR (2001) Computer versus lecture: a comparison of two methods of teaching oral medication administration in a nursing skills laboratory. J Nurs Educ 40:323-329

119. Jeffries PR, Woolf S, Linde B (2003) Technologybased vs. traditional instruction. A comparison of two methods for teaching the skill of performing a 12-lead ECG. Nurs Educ Perspect 24:70-74

120. Miller SW, Jackson RA (1985) A comparison of a multi-media instructional module with a traditional lecture format for geriatric pharmacy training. Am J Pharm Educ 49:173-176

121. O'Leary S, Diepenhorst L, Churley-Strom R, Magrane D (2005) Educational games in an obstetrics and gynecology core curriculum. Am J Obstet Gynecol 193:1848-1851

122. Ryan G, Lyon P, Kumar K et al (2007) Online CME: an effective alternative to face-to-face delivery. Med Teach 29:e251-e257

123. Schlomer RS, Anderson MA, Shaw R (1997) Teaching strategies and knowledge retention. J Nurs Staff Dev 13:249-253

124. Perkins GD (2007) Simulation in resuscitation training. Resuscitation 73:202-211

125. Campbell DM, Barozzino T, Farrugia M, Sgro M (2009) High-fidelity simulation in neonatal resuscitation. Paediatr Child Health 14:19-23

126. Donoghue AJ, Durbin DR, Nadel FM et al (2009) Effect of highfidelity simulation on Pediatric Advanced Life Support training in pediatric house staff: a randomized trial. Pediatr Emerg Care 25:139-144

127. Mayo PH, Hackney JE, Mueck JT et al (2004) Achieving house staff competence in emergency airway management: results of a teaching program using a computerized patient simulator. Crit Care Med 32:2422-2427

128. Owen H, Mugford B, Follows V, Plummer JL (2006) Comparison of three simulation-based training methods for management of medical emergencies. Resuscitation 71:204-211

129. Wayne DB, Butter J, Siddall VJ et al (2005) Simulation-based training of internal medicine residents in advanced cardiac life support protocols: a randomized trial. Teach Learn Med 17:210-216
130. Ali J, Cohen RJ, Gana TJ, Al-Bedah KF (1998) Effect of the Advanced Trauma Life Support program on medical students' performance in simulated trauma patient management. J Trauma 44:588-591

131. Hunt EA, Vera K, Diener-West M et al (2009) Delays and errors in cardiopulmonary resuscitation and defibrillation by pediatric residents during simulated cardiopulmonary arrests. Resuscitation 80:819-825

132. Rodgers D, Securro SJ, Pauley R (2009) The Effect of High-Fidelity Simulation on Educational Outcomes In An Advanced Cardiovascular Life Support Course. Simul Healthc 4:200-206

133. Barsuk D, Ziv A, Lin G et al (2005) Using advanced simulation for recognition and correction of gaps in airway and breathing management skills in prehospital trauma care. Anesth Analg 100:803809 , table of contents

134. Kory PD, Eisen LA, Adachi M et al (2007) Initial airway management skills of senior residents: simulation training compared with traditional training. Chest 132:1927-1931

135. Marshall RL, Smith JS, Gorman PJ et al (2001) Use of a human patient simulator in the development of resident trauma management skills. J Trauma 51:17-21

136. Wayne DB, Siddall VJ, Butter J et al (2006) A longitudinal study of internal medicine residents' retention of advanced cardiac life support skills. Acad Med 81:S9-S12

137. Cherry RA, Williams J, George J, Ali J (2007) The effectiveness of a human patient simulator in the ATLS shock skills station. J Surg Res 139:229-235

138. Curran VR, Aziz K, O'Young S, Bessell C (2004) Evaluation of the effect of a computerized training simulator (ANAKIN) on the retention of neonatal resuscitation skills. Teach Learn Med 16:157-164

139. Friedman Z, You-Ten KE, Bould MD, Naik V (2008) Teaching lifesaving procedures: the impact of model fidelity on acquisition and transfer of cricothyrotomy skills to performance on cadavers. Anesth Analg 107:1663-1669

140. Hoadley TA (2009) Learning advanced cardiac life support: a comparison study of the effects of lowand high-fidelity simulation. Nurs Educ Perspect 30:91-95

141. Iglesias-Vazquez JA, Rodriguez-Nunez A, PenasPenas M et al (2007) Cost-efficiency assessment of Advanced Life Support (ALS) courses based on the comparison of advanced simulators with conventional manikins. BMC Emerg Med 7:18

142. Schwartz LR, Fernandez R, Kouyoumjian SR et al (2007) A randomized comparison trial of casebased learning versus human patient simulation in medical student education. Acad Emerg Med 14:130-137

143. Wang XP, Martin SM, Li YL et al (2008) Effect of emergency care simulator combined with problem-based learning in teaching of cardiopulmonary resuscitation. Zhonghua Yi Xue Za Zhi 88:1651-1653

144. Pottle A, Brant S (2000) Does resuscitation training affect outcome from cardiac arrest? Accid Emerg Nurs 8:46-51

145. Birnbaum ML, Robinson NE, Kuska BM et al (1994) Effect of advanced cardiac life-support training in rural, community hospitals. Crit Care Med 22:741-749 
146. Makker R, Gray-Siracusa K, Evers M (1995) Evaluation of advanced cardiac life support in a community teaching hospital by use of actual cardiac arrests. Heart Lung 24:116-120

147. Schneider T, Mauer D, Diehl P et al (1995) Does standardized mega-code training improve the quality of pre-hospital advanced cardiac life support (ACLS)? Resuscitation 29:129-134

148. Bruppacher HR, Alam SK, LeBlanc VR et al (2010) Simulation-based training improves physicians' performance in patient care in high-stakes clinical setting of cardiac surgery. Anesthesiology 112:985-992

149. Wayne DB, Didwania A, Feinglass J et al (2008) Simulation-based education improves quality of care during cardiac arrest team responses at an academic teaching hospital: a case-control study. Chest 133:56-61

150. Cavaleiro AP, Guimaraes H, Calheiros F (2009) Training neonatal skills with simulators? Acta Paedia$\operatorname{tr} 98: 636-639$

151. Knudson MM, Khaw L, Bullard MK et al (2008) Trauma training in simulation: translating skills from SIM time to real time. J Trauma 64:255-263; discussion 63-64

152. Miotto HC, Couto BR, Goulart EM et al (2008) Advanced Cardiac Life Support Courses: live actors do not improve training results compared with conventional manikins. Resuscitation 76:244-248

153. Ali J, Al Ahmadi K, Williams Jl, Cherry RA (2009) The standardized live patient and mechanical patient models - their roles in trauma teaching. J Trauma 66:98-102

154. Mueller MP, Christ T, Dobrev D et al (2005) Teaching antiarrhythmic therapy and ECG in simulatorbased interdisciplinary undergraduate medical education. Br J Anaesth 95:300-304

155. Kobayashi L, Lindquist DG, Jenouri IM et al (2010) Comparison of sudden cardiac arrest resuscitation performance data obtained from in-hospital incident chart review and in situ high-fidelity medical simulation. Resuscitation 81:463-471

156. Edelson DP, Eilevstjonn J, Weidman EK et al (o J) Capnography and chest-wall impedance algorithms for ventilation detection during cardiopulmonary resuscitation. Resuscitation 81:317-322

157. Duran R, Aladag N, Vatansever U et al (2008) Proficiency and knowledge gained and retained by pediatric residents after neonatal resuscitation course. Pediatr Int 50:644-647

158. Anthonypillai $F$ (1992) Retention of advanced cardiopulmonary resuscitation knowledge by intensive care trained nurses. Intensive Crit Care Nurs 8:180-184

159. Boonmak P, Boonmak S, Srichaipanha S, Poomsawat S (2004) Knowledge and skill after brief ACLS training. J Med Assoc Thai 87:1311-1314

160. Kaye W, Wynne G, Marteau T et al (1990) An advanced resuscitation training course for preregistration house officers. J R Coll Physicians Lond 24:51-54

161. Semeraro F, Signore L, Cerchiari EL (2006) Retention of CPR performance in anaesthetists. Resuscitation 68:101-108

162. Skidmore MB, Urquhart $H$ (2001) Retention of skills in neonatal resuscitation. Paediatr Child Health 6:31-35
163. Trevisanuto D, Ferrarese $P$, Cavicchioli $P$ et al (2005) Knowledge gained by pediatric residents after neonatal resuscitation program courses. $\mathrm{Pa}$ ediatr Anaesth 15:944-947

164. Young R, King L (2000) An evaluation of knowledge and skill retention following an in-house advanced life support course. Nurs Crit Care 5:714

165. Grant EC, Marczinski CA, Menon K (2007) Using pediatric advanced life support in pediatric residency training: does the curriculum need resuscitation? Pediatr Crit Care Med 8:433-439

166. O'Steen DS, Kee CC, Minick MP (1996) The retention of advanced cardiac life support knowledge among registered nurses. J Nurs Staff Dev 12:6672

167. Hammond F, Saba M, Simes T, Cross R (2000) Advanced life support: retention of registered nurses' knowledge 18 months after initial training. Aust Crit Care 13:99-104

168. Nadel FM, Lavelle JM, Fein JA et al (2000) Assessing pediatric senior residents' training in resuscitation: fund of knowledge, technical skills, and perception of confidence. Pediatr Emerg Care 16:73-76

169. Napier F, Davies RP, Baldock C et al (2009) Validation for a scoring system of the ALS cardiac arrest simulation test (CASTest). Resuscitation 80:10341038

170. White JR, Shugerman R, Brownlee C, Quan L (1998) Performance of advanced resuscitation skills by pediatric housestaff. Arch Pediatr Adolesc Med 152:1232-1235

171. Rodgers DL, Bhanji F, McKee BR (2010) Written evaluation is not a predictor for skills performance in an advanced cardiovascular life support course. Resuscitation 81:453-456

172. Kromann CB, Jensen ML, Ringsted C (2009) The effect of testing on skills learning. Med Educ 43:21-27

173. Kromann CB, Bohnstedt C, Jensen ML, Ringsted $C$ (2009) The testing effect on skills learning might last 6 months. Adv Health Sci Educ Theory Pract

174. Choa M, Park I, Chung HS et al (2008) The effectiveness of cardiopulmonary resuscitation instruction: animation versus dispatcher through a cellular phone. Resuscitation 77:87-94

175. Choa M, Cho J, Choi YH et al (2009) Animation-as sisted CPRIl program as a reminder tool in achieving effective one-person-CPR performance. Resuscitation 80:680-684

176. Ertl L, Christ F (2007) Significant improvement of the quality of bystander first aid using an expert system with a mobile multimedia device. Resuscitation 74:286-295

177. Ward P, Johnson LA, Mulligan NW et al (1997) Improving cardiopulmonary resuscitation skills retention: effect of two checklists designed to prompt correct performance. Resuscitation 34:221-225

178. Berkenstadt $H$, Yusim Y, Ziv A et al (2006) An assessment of a point-of-care information system for the anesthesia provider in simulated malignant hyperthermia crisis. Anesth Analg 102:530-532

179. Lerner C, Gaca AM, Frush DP et al (2009) Enhancing pediatric safety: assessing and improving resident competency in life-threatening events with a computer-based interactive resuscitation tool. Pediatr Radiol 39:703-709
180. Schneider AJ, Murray WB, Mentzer SC et al (1995) Helper: A critical events prompter for unexpected emergencies. J Clin Monit 11:358-364

181. Dyson E, Voisey S, Hughes S et al (2004) Educational psychology in medicallearning: a randomised controlled trial of two aide memoires for the recall of causes of electromechanical dissociation. Emerg Med J 21:457-460

182. McCallum Z, South M (2004) Development and use of a portable paediatric resuscitation card. J Paediatr Child Health 40:477-480

183. Mills PD, DeRosier JM, Neily J et al (2004) A cognitive aid for cardiac arrest: you can't use it if you don't know about it. Jt Comm J Qual Saf 30:488496

184. Neily J, DeRosier JM, Mills PD et al (2007) Awareness and use of a cognitive aid for anesthesiology. Jt Comm J Qual Patient Saf 33:502-511

185. Zanner R, Wilhelm D, Feussner H, Schneider G (2007) Evaluation of M-AID, a first aid application for mobile phones. Resuscitation 74:487-494

186. Nelson KL, Shilkofski NA, Haggerty JA et al (2008) The use of cognitive AIDS during simulated pediatric cardiopulmonary arrests. Simul Healthc 3:138-145

187. Mikrogianakis A, Osmond MH, Nuth JE et al (2008) Evaluation of a multidisciplinary pediatric mock trauma code educational initiative: a pilot study. J Trauma 64:761-77

188. Farah R, Stiner E, Zohar Z et al (2007) Cardiopulmonary resuscitation surprise drills for assessing, improving and maintaining cardiopulmonary resuscitation skills of hospital personnel. Eur J Emerg Med 14:332-336

189. Cappelle C, Paul RI (1996) Educating residents: the effects of a mock code program. Resuscitation 31:107-111

190. Villamaria FJ, Pliego JF, Wehbe-Janek $\mathrm{H}$ et al (2008) Using simulation to orient code blue teams to a new hospital facility. Simul Healthc 3:209-216

191. Hunt EA, Hohenhaus SM, Luo X, Frush KS (2006) Simulation of pediatric trauma stabilization in 35 North Carolina emergency departments: identification of targets for performance improvement. Pediatrics 117:641-648

192. Hunt EA, Walker AR, Shaffner DH et al (2008) Simulation of in-hospital pediatric medical emergencies and cardiopulmonary arrests: highlighting the importance of the first 5 minutes. Pediatrics 121:e34-e43

193. Pittman J, Turner B, Gabbott DA (2001) Communication between members of the cardiac arrest team - a postal survey. Resuscitation 49:175-177

194. Morgan R, Westmoreland C (2002) Survey of junior hospital doctors' attitudes to cardiopulmonary resuscitation. Postgrad Med J 78:413-415

195. Savoldelli GL, Naik VN, Park J et al (2006) Value of debriefing during simulated crisis management: oral versus video-assisted oral feedback. Anesthesiology 105:279-285

196. Clay AS, Que L, Petrusa ER et al (2007) Debriefing in the intensive care unit: a feedback tool to facilitate bedside teaching. Crit Care Med 35:738-754

197. Dine CJ, Gersh RE, Leary M et al (2008) Improving cardiopulmonary resuscitation quality and resuscitation training by combining audiovisual feedback and debriefing. Crit Care Med 36:28172822 


\section{In eigener Sache}

198. Falcone RA Jr, Daugherty M, Schweer L et al (2008) Multidisciplinary pediatric trauma team training using high-fidelity trauma simulation. J Pediatr Surg 43:1065-1071

199. Goffman D, Heo H, Pardanani S et al (2008) Improving shoulder dystocia management among resident and attending physicians using simulations. Am J Obstet Gynecol 199:294 e1-e5

200. Hoyt DB, Shackford SR, Fridland PH et al (1988) Video recording trauma resuscitations: an effective teaching technique. J Trauma 28:435-440

201. Morgan PJ, Tarshis J, LeBlanc V et al (2009) Efficacy of high-fidelity simulation debriefing on the performance of practicing anaesthetists in simulated scenarios. Br J Anaesth 103:531-537

202. Pope C, Smith A, Goodwin D, Mort M (2003) Passing on tacit knowledge in anaesthesia: a qualitative study. Med Educ 37:650-655

203. Scherer LA, Chang MC, Meredith JW, Battistella FD (2003) Videotape review leads to rapid and sustained learning. Am J Surg 185:516-520

204. Townsend RN, Clark R, Ramenofsky ML, Diamond DL (1993) ATLS-based videotape trauma resuscitation review: education and outcome. J Trauma 34:133-138

205. Weng TI, Huang CH, Ma MH et al (2004) Improving the rate of return of spontaneous circulation for out-of-hospital cardiac arrests with a formal, structured emergency resuscitation team. Resuscitation 60:137-142

206. Baskett PJ, Lim A (2004) The varying ethical attitudes towards resuscitation in Europe. Resuscitation 62:267-273

207. Sandroni C, Fenici P, Cavallaro F et al (2005) Haemodynamic effects of mental stress during cardiac arrest simulation testing on advanced life support courses. Resuscitation 66:39-44

208. Soar J, Perkins GD, Harris S et al (2003) The immediate life support course. Resuscitation 57:21-26

209. Soar J, McKay U (1998) A revised role for the hospital cardiac arrest team? Resuscitation 38:145149

210. Smith GB, Osgood VM, Crane S (2002) ALERT - a multiprofessional training course in the care of the acutely ill adult patient. Resuscitation 52:281286

211. Spearpoint KG, Gruber PC, Brett SJ (2009) Impact of the Immediate Life Support course on the incidence and outcome of in-hospital cardiac arrest calls: an observational study over 6 years. Resuscitation 80:638-643

212. Nolan J (2001) Advanced life support training. Resuscitation 50:9-11

213. Perkins G, Lockey A (2002) The advanced life support provider course. BMJ 325:S81

214. Ringsted C, Lippert F, Hesselfeldt R et al (2007) Assessment of Advanced Life Support competence when combining different test methods - reliability and validity. Resuscitation 75:153-160

215. Perkins GD, Davies RP, Stallard N et al (2007) Advanced life support cardiac arrest scenario test evaluation. Resuscitation 75:484-490

216. Buss PW, McCabe M, Evans RJ et al (1993) A survey of basic resuscitation knowledge among resident paediatricians. Arch Dis Child 68:75-78

217. Carapiet D, Fraser J, Wade A et al (2001) Changes in paediatric resuscitation knowledge among doctors. Arch Dis Child 84:412-414

\section{www.SpringerMedizin.de Das Internet der Ärzte}

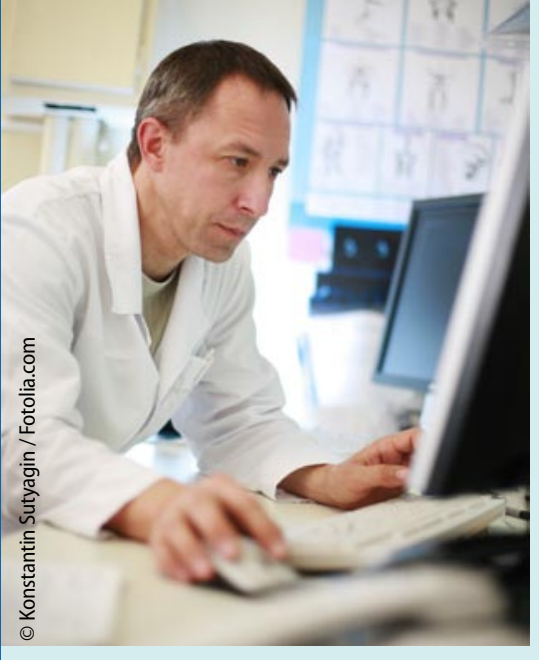

Sie möchten auf dem Laufenden bleiben? In umfassenden Zeitschriftenbibliotheken recherchieren? Sich fortbilden und CME-Punkte sammeln oder bei Experten Ihres Fachgebiets Rat suchen? Dann sind Sie auf SpringerMedizin.de, dem neuen Fachportal für Ärzte, goldrichtig. Denn dort erwartet Sie eine Fülle von nützlichen Informationen - maßgeschneidert auf Ihr Fachgebiet.
Titeln geordnet. In dieser umfassenden Online-Bibliothek stehen für Abonnenten die Inhalte von über 70 renommierten Titeln bis in die 1990er-Jahre bereit.

Ihr personalisiertes Informationsangebot

SpringerMedizin.de ist ein Fachportal für Ärzte, daher sind alle medizinischen Inhalte passwortgeschützt. Die kostenlose Registrierung ist schnell erledigt und hat einen großen Vorteil: Sobald Sie bei der Registrierung $\mathrm{Ihr}$ Fachgebiet angegeben haben, erhalten Sie künftig automatisch als personalisierte Startseite das entsprechende Fachportal angezeigt. Ihre Startseite können Sie nach Wunsch weiter anpassen: Die grauen Rubrikenkästen (sog. Portlets) lassen sich verschieben, umgruppieren oder auch wegklicken. Über den Knopf „Mehr Inhalte hinzufügen" können Sie weitere Rubriken auswählen und auf der Startseite anzeigen lassen. Möchten Sie auch News und Beiträge aus anderen Fachgebieten lesen - kein Problem, konfigurieren Sie Ihr Portlet ganz

\section{Von Anaesthesie bis Zahnmedizin - die ganze Welt der Medizin ist nur wenige Klicks entfernt. www.SpringerMedizin.de ist das Fachportal für Ärzte in Deutschland}

Es lohnt sich, mehrmals am Tag mit SpringerMedizin.de online zu gehen. Täglich stellt Innen das Redaktionsteam neue interessante Themen aus Medizin und Gesundheitspolitik zusammen. Weitere News aus der Hauptstadt und Informationen, was sich in den einzelnen Bundesländern und KVen tut, stehen im Rubrikenkasten "Gesundheitspolitik" bereit. Täglich neue Meldungen zu medizinischen Themen finden Sie unter "Medizin kompakt". Kurz und bündig geben Ihnen diese Meldungen einen schnellen Überblick, was sich in der Medizin alles tut.

\section{Fortbildung und $\mathrm{CME}$}

Unter dem Navigationspunkt "Fortbildung" stehen Ihnen interessante Übersichtsarbeiten, spannende Kasuistiken und CMEBeiträge aus den Springer-Fachzeitschriften zur Verfügung. Unter "Zeitschriften“ finden Sie das gesamte Zeitschriftenangebot von Springer Medizin nach Fachgebieten und nach Wunsch. Sobald Sie die Konfiguration abgespeichert haben, erscheint sie beim nächsten Log-in genau so wieder. Über den orangenen Button „"Guided Tour" erklären wir Ihnen alle Möglichkeiten der individuellen Anpassung in einem Film.

Diese Beispiele sind nur ein Bruchteil dessen, was SpringerMedizin. de zu bieten hat. Steuern Sie das Internet der Ärzte an und lassen Sie sich überraschen! Es lohnt sich.

\section{Dr. Sonja Kempinski Chefredakteurin SpringerMedizin.de}

